\title{
OPEN Investigation of the transcriptomic response in Atlantic salmon (Salmo salar) gill exposed to Paramoeba perurans during early onset of disease
}

\author{
Anita Talbot ${ }^{1 凶}$, Laura Gargan², Grainne Moran ${ }^{1}$, Louis Prudent ${ }^{1}$, Ian O'Connor $^{1}$, \\ Luca Mirimin ${ }^{1}$, Jens Carlsson ${ }^{2}$ \& Eugene MacCarthy ${ }^{1}$
}

Amoebic Gill Disease (AGD), caused by the protozoan extracellular parasite Paramoeba perurans ( $P$. perurans) is a disease affecting Atlantic salmon (Salmo salar). This study investigated the gill transcriptomic profile of pre-clinical AGD using RNA-sequencing (RNA-seq) technology. RNA-seq libraries generated at 0, 4, 7, 14 and 16 days post infection (dpi) identified 19,251 differentially expressed genes (DEGs) of which $56.2 \%$ were up-regulated. DEGs mapped to 224 Gene Ontology (GO) terms including 140 biological processes (BP), 45 cellular components (CC), and 39 molecular functions (MF). A total of 27 reference pathways in the Kyoto Encyclopedia of Genes and Genomes (KEGG) and 15 Reactome gene sets were identified. The RNA-seq data was validated using real-time, quantitative PCR (qPCR). A host immune response though the activation of complement and the acute phase genes was evident at $7 \mathrm{dpi}$, with a concurrent immune suppression involving cytokine signalling, notably in interleukins, interferon regulatory factors and tumour necrosis factor-alpha (tnf- $\alpha$ ) genes. Down-regulated gene expression with involvement in receptor signalling pathways (NOD-like, Tolllike and RIG-1) were also identified. The results of this study support the theory that $P$. perurans can evade immune surveillance during the initial stages of gill colonisation through interference of signal transduction pathways.

Amoebic Gill Disease (AGD) is a gill disease of marine cultured Atlantic salmon, caused by the free-living protozoan Paramoeba perurans (P. perurans) ${ }^{1}$ also known as Neoparamoeba perurans. Among the parasitic conditions affecting gill health, AGD is the most significant in terms of prevalence and economic impact ${ }^{2}$. First described in Tasmania in $1985^{1}$, AGD is now present in most Atlantic salmon producing countries ${ }^{2}$ and affects fish during the marine grow out cycle of production at sea temperatures ranging from 7 to $20^{\circ} \mathrm{C}^{2}$. Macroscopically, AGD lesions are visible as white raised mucoid patches on the gill, due to increased mucus production by mucous cells. The colonisation of the gills by $P$. perurans initiates a tri-phasic host response which includes a localised reaction to parasite attachment, non-specific immuno-regulatory cell infiltration and advanced hyperplasia with epithelial stratification $^{3}$. Proliferation and fusion of the lamellar epithelium decreases the functional gill surface area and increases the diffusion distance in the water-blood barrier for oxygen transfer. Consequently, AGD-affected individuals experience respiratory disturbances ${ }^{4}$. Microscopically, fresh gill smears can be used to detect the presence of amoebae. Additionally, histological examination of AGD gill tissue is used to confirm hyperplasia, lamellar fusion, the presence of lamellar vesicles in addition to the presence of amoeba, while species identification can be confirmed using a real-time PCR ( $\mathrm{qPCR}$ ) assay ${ }^{5}$. A widely used practical method for determining the presence and severity of AGD is the gross gill score ${ }^{6}$ ( 0 indicating no AGD, and 5 signifying extensive disease) across each side of all 8 gills. In the absence of a vaccine, current AGD treatments are limited to freshwater or hydrogen peroxide baths, which remove the amoebae from affected gills, and reduce gill mucus viscosity ${ }^{3,7}$. Bath treatments can cause stress and result in mortalities, and multiple treatments may be required during the grow-out production cycle, due to high re-infection rates. Gill scoring is the primary method employed to monitor AGD and is often used to initiate treatment. However, a recent study looking at gene expression, gross morphology

${ }^{1}$ Galway Mayo Institute of Technology, Galway, Ireland. ${ }^{2}$ University College Dublin, Dublin 4, Ireland. ${ }^{\square}$ email: anita.talbot@gmit.ie 
and histopathology as tools for monitoring proliferate gill disease (PGD) in salmon reported a lack of association between gill score and gene expression or histology ${ }^{8}$. Previous AGD gill gene expression studies have utilised numerous molecular techniques including microarray analysis ${ }^{9-11}$, polymerase chain reaction (PCR $)^{12-17}$, and more recently RNA-seq ${ }^{18,19}$ to investigate host response expression profiles. Although results can vary depending on the dose and virulence of the P. perurans, fish size, duration of infection and use of lesion or non-lesion specific gill tissue, these earlier studies found a localised immune suppression with genes involved in Th1, Th17 and Tregs pathways being down-regulated ${ }^{12}$, while pro-inflammatory cytokines (Il-1 $\left.\beta\right)^{13}$, and Th2 cytokines (il4/13a and il4/13b2) $)^{12,14}$ were up-regulated. Mucus production is increased with AGD and investigation into mucin genes expression found mucin 5 to be up-regulated ${ }^{14}$. The differential expression of the superoxide dismutase and catalase genes involved in oxidative stress has also been reported in late stage AGD ${ }^{20}$. The down-regulation of tumour suppressor p53 (p53) has been proposed as one of the mechanisms underlying cell proliferation in $\mathrm{AGD}^{21}$. Resistance to AGD has a moderate heritable genetic component and a recent study combining genome wide association analysis with RNA-seq gene expression profiling in the gill, reported the down-regulation of immune system components during the initial stages of the infection, while genes involved in cell adhesion processes were found to be up-regulated ${ }^{18}$. The involvement of the interferon response, systemic inflammation and apoptosis have also been associated with AGD resistance ${ }^{22}$. More recently, a dual RNA-seq study investigating the host-parasite interaction identified two transcription factors, znfOZF-like and znf70-like, with involvement in the immune response, cellular proliferation and invasion to be differentially expressed in response to AGD. The aim of this study was to utilise RNA-seq technology to investigate further the gill transcriptome, subsequent to P. perurans colonisation but in advance of mucoid lesion development, in AGD-affected fish, in order to identify biomarkers of early-stage disease.

\section{Results}

Gill score. Clinical symptoms of AGD were determined by macroscopic examination of the intact gills in euthanised fish and were scored according to Taylor et al. ${ }^{6}$. Prior to sampling, 3 fish were taken from each duplicate control and AGD-affected tanks, and all 6 fish placed in an anaesthetic bath. Naïve control fish (6) and experimental fish (6) were sampled at each time point (0, 4, 7, 14 and $16 \mathrm{dpi})$ and all fish were gill scored. No lesions were evident on the gills in AGD-affected groups at 4 and $7 \mathrm{dpi}$, with $3 / 6$ fish at gill score 1 at $14 \mathrm{dpi}$. At $16 \mathrm{dpi}$, all six fish had a gill score of between 1 and 2 .

Detection of $P$. perurans DNA. Gill tissue from six naïve control, and six AGD-affected fish were analysed by qPCR for the presence of $P$. perurans DNA at $0,4,7,14$ and 16 dpi. The higher the crossing threshold value $(\mathrm{Ct})$, the less $P$. perurans DNA that was detected. The qPCR was negative for the naïve control fish at all five sampling time points. The Ct values in the AGD-affected fish at 4 dpi were $34.6 \pm 0.47, \mathrm{n}=5$ ( 1 fish had a negative result with no $P$. perurans DNA detected), at $7 \mathrm{dpi}$ was $\mathrm{Ct} 31.8 \pm 2.7, \mathrm{n}=6$, at $14 \mathrm{dpi}$ the $\mathrm{Ct}$ was $28.8+1.5$, and at 16 dpi the Ct was $29.4+1.7$. The qPCR results indicate an increasing amoebic load, based on the level of $P$. perurans DNA detected, on the gill from 4 to $16 \mathrm{dpi}$.

Histopathology analysis. Gills were examined microscopically following staining with haematoxylin/ eosin (Fig. 1). Some small areas of mild epithelial hyperplasia was evident at $4 \mathrm{dpi}$, while more extensive hyperplasia and lamellar fusion can be seen at 7dpi. Substantial hyperplasia and lamellar fusion with the formation of lamellar vesicles, indicative of AGD, were evident at $14 \mathrm{dpi}$ and $16 \mathrm{dpi}$.

Mapping RNA-seq reads to reference genome. Information related to the number of reads and alignments to the Salmo salar reference genome ICSASG_v2 (GenBank: GCF_000233375.1) $)^{23}$ for the 6 fish from each of the 5 sampling time points $(0,4,7,14$ and $16 \mathrm{dpi})$ is presented in Supplementary Table $\mathrm{S} 1$ online. The sequencing depth ranged from 21.7 to $35.4 \mathrm{M}$ raw reads. The number of clean, paired reads ranged from 19.8 to $32.9 \mathrm{M}$ ( $88.4 \%$ to $94.7 \%)$. Assigned fragments in HISAT2 ranged from 18.7 to $31.8 \mathrm{M}$ (91\% to $97 \%$ ) and the number of assigned feature counts ranged from 14.7 to $25.2 \mathrm{M}$.

Sample similarity. Principal component analysis (PCA) was generated to visualise the relationships between all samples, as well as samples by time point (Supplementary Fig. S1 online). The similarity between samples from thirty-six RNA-seq libraries ( 6 fish $\times 6$ groups, including 2 groups from T0) was analysed. Only one of the control libraries (T0_GCRL) was used in the pairwise comparisons. The variation in the PC1 was $70 \%$, and in PC2 was 4\%. From the PCA plot, it was evident that four of the six AGD-affected samples at 7 dpi clustered together, while one sample aligned with the earlier time points, and one sample aligning with the later time points.

Differentially expressed genes (DEGs). Differentially expressed genes were identified at each of the four experimental time points $(4,7,14$ and $16 \mathrm{dpi})$ relative to naïve control fish $(0 \mathrm{dpi})$. The number of genes differentially regulated in the AGD-affected gill tissue was 19,251 of which $56.2 \%$ were up-regulated over the course of the AGD trial. The pattern of gene expression at 4,14 and 16 dpi identified more genes down-regulated $\left(61.6 \%, 52.4 \%\right.$, and $54.7 \%$ respectively) with the majority of genes having a $\log _{2}$ fold change $\left(\log _{2} \mathrm{FC}\right)$ expression between -2 and +2 . The pattern of gene expression shifted more toward gene up-regulation at 7 dpi $(59.6 \%$ vs $40.4 \%)$ and a greater increase in the $\log _{2}$ fold expression with most genes falling between a $\log _{2} \mathrm{FC}$ of -2 and +15 (Fig. 2). 

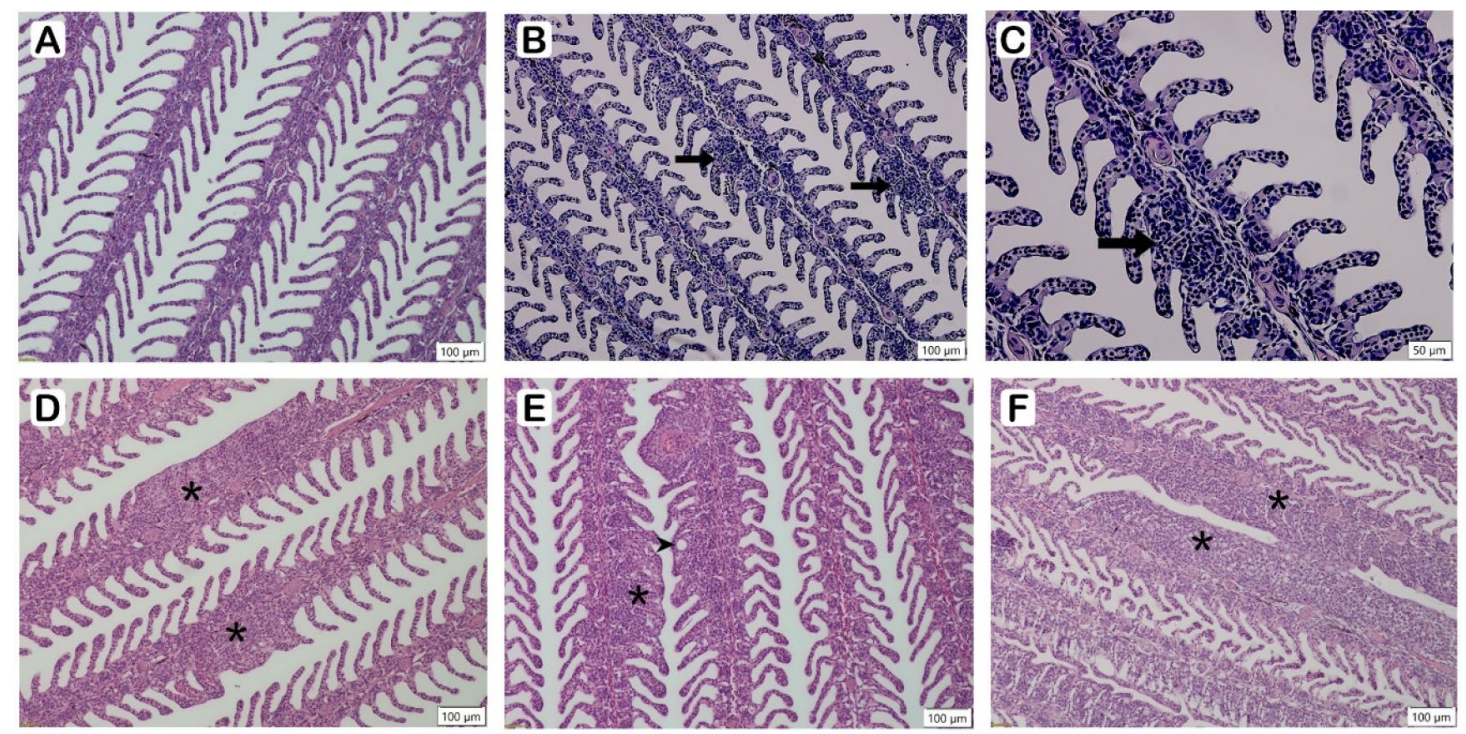

Figure 1. Histology in AGD-affected gills at 0 dpi, (A) 4 dpi, (B,C) 7 dpi, (D) 14 dpi, (E) and 16 dpi (F) in AGD-affected gill tissue. Normal gill naïve control fish showing individual gill filaments with lamellae (A). Some small areas of mild epithelial hyperplasia is evident at $4 \mathrm{dpi}$, indicated by arrows, (B), also shown at a higher magnification (C). More extensive hyperplasia and lamellar fusion, indicative of AGD, can be seen at 7dpi (D) and is indicated by asterisks. The increasing hyperplasia, lamellar fusion (asterisks) with the formation of lamellar vesicles (arrowhead) is evident at $14 \mathrm{dpi}(\mathbf{E})$. At $16 \mathrm{dpi}(\mathbf{F})$ there is extensive lamellae fusion in adjoining gill filaments (asterisks). Slides were stained with H\&E and the scale is indicated on each image.
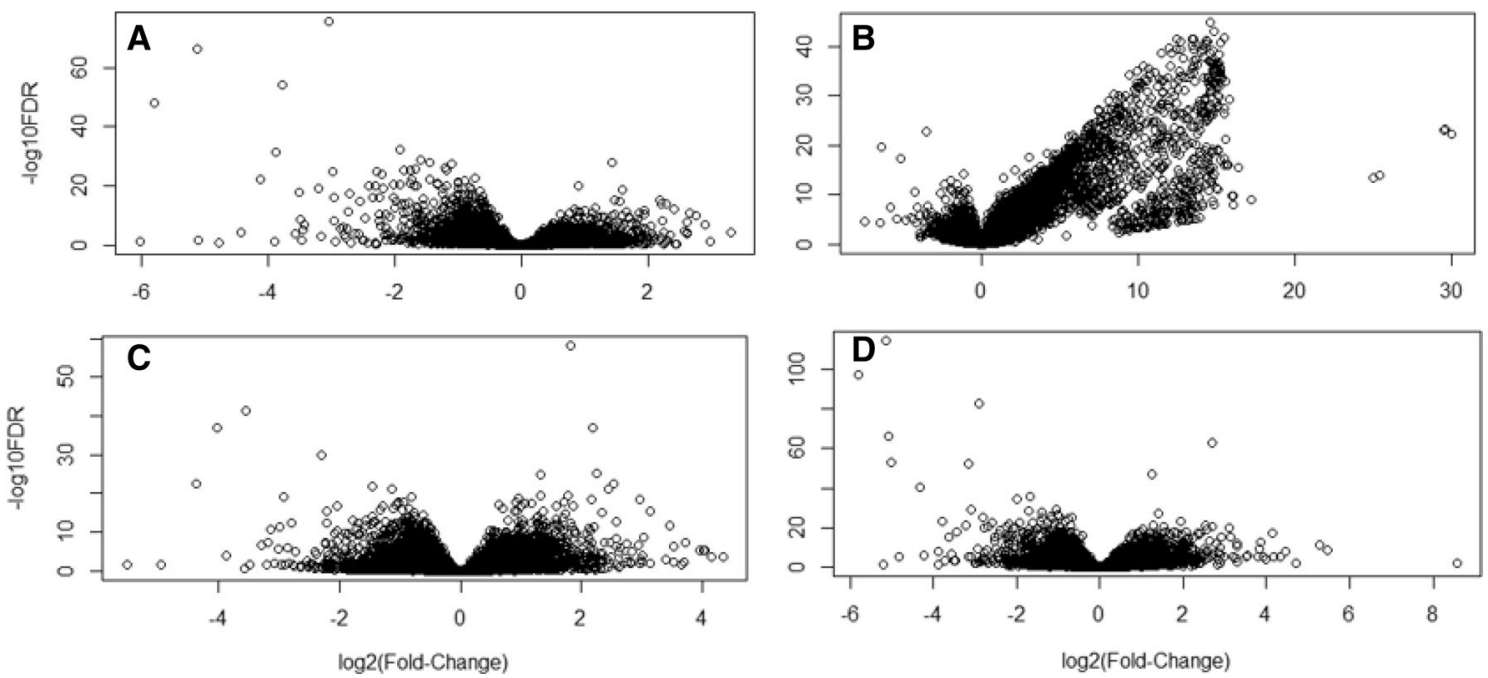

Figure 2. Volcano plots illustrating the pattern of differential gene expression over time. Plots represent $4 \mathrm{dpi}$ (A), $7 \mathrm{dpi}$ (B), 14dpi (C) and $16 \mathrm{dpi}$ (D). The $\mathrm{X}$-axis represents the $\log _{2} \mathrm{FC}$ in expression with negative numbers representing down-regulated expression and positive numbers representing up-regulated expression. The $\mathrm{Y}$-axis is the $p$-value adjusted for or the false discovery rate $(\mathrm{FDR}<0.05)$ to the negative log base 10 . The pattern of expression was different at $7 \mathrm{dpi}$ with more genes being differentially expressed, more genes being upregulated and with a higher $\log _{2} \mathrm{FC}$ compared to the other time-points.

Of the total number of differentially regulated genes (Salmo salar gene IDs) identified by RNA-seq, 8425 were down-regulated and 10,826 up-regulated. A number of genes were differentially expressed at only one time point; 4 dpi; 1110 genes with 662 down- and 448 up-regulated, 7 dpi; 9469 genes with 3107 down- and 6362 upregulated, 14 dpi; 1163 genes with 506 down- and 657 up-regulated, 16 dpi; 1240 genes with 588 down- and 652 up-regulated. There were 609 down-regulated genes and 210 up-regulated common to all four time points (Fig. 3).

Validation of RNA-seq data using qPCR. Ten genes from the gill RNA-seq data were selected for qPCR validation across all sampling time points (Table 1). All analysis was carried out on individual fish gill samples $(\mathrm{n}=30)$. The qPCR pairwise comparisons matched those carried out in the RNA-seq study. Genes included acod1: aconitate decarboxylase 1 (also known as irg1), cath1: cathelicidin 1, clra: c type lectin receptor a, cc4: $c-c$ 

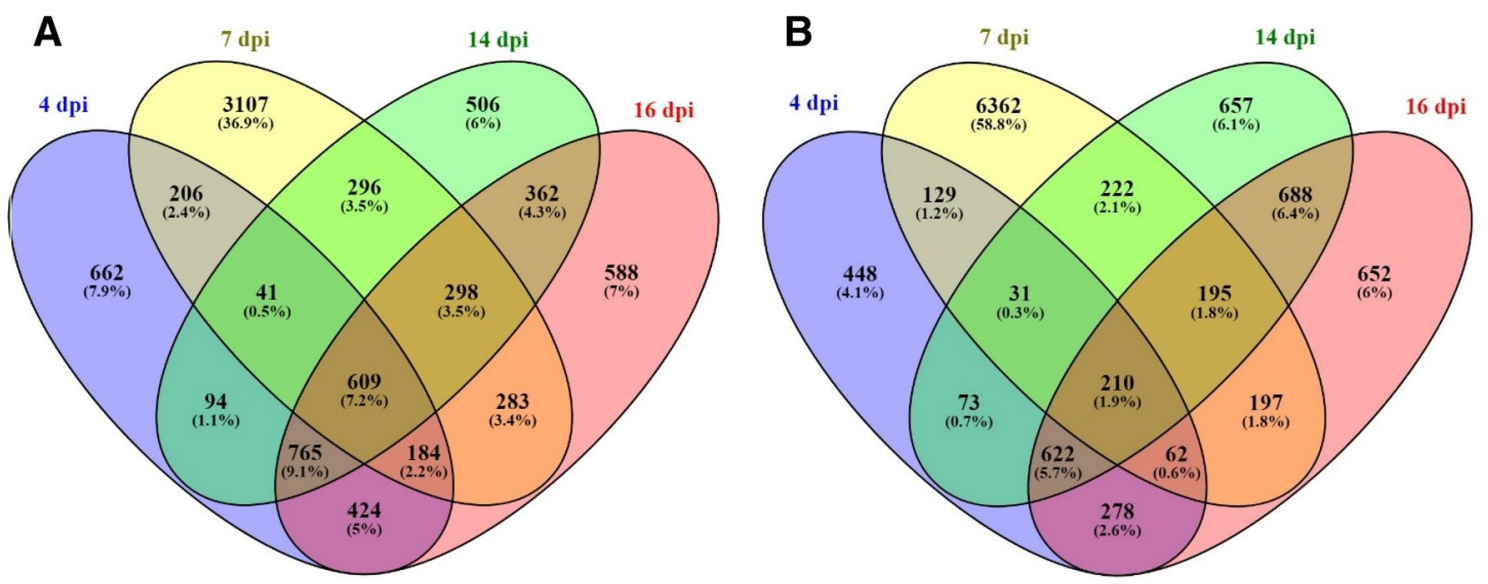

Figure 3. The distribution of differentially expresses genes in AGD-affected gill. The four-way Venn diagrams show the number and percentage of genes (A) down-regulated and (B) up-regulated at 4, 7, 14 and 16 dpi. Genes with a $p$-value adjusted for a false discovery rate $(\mathrm{FDR})<0.05$ were assigned as differentially expressed in DESeq.

\begin{tabular}{|c|c|c|c|c|c|c|c|c|c|}
\hline \multirow[b]{2}{*}{ Gene ID } & \multirow[b]{2}{*}{ Description } & RNA-seq & qPCR & RNA-seq & qPCR & RNA-seq & qPCR & RNA-seq & qPCR \\
\hline & & \multicolumn{2}{|l|}{ FC, 4 dpi } & \multicolumn{2}{|l|}{ FC, 7 dpi } & \multicolumn{2}{|c|}{ FC, 14 dpi } & \multicolumn{2}{|c|}{ FC, 16 dpi } \\
\hline 106581616 & acod & -7.7 & -7.1 & -8.5 & -12.7 & -6.9 & -9 & -20.2 & -22.3 \\
\hline 100136453 & cath1 & -7.7 & -5.9 & -9.1 & -9.0 & nd & 1.1 & -2.5 & -2.5 \\
\hline 100136446 & clra & -4.7 & -1.4 & 34 & 75.9 & -4.6 & 1.1 & -7.1 & -1.4 \\
\hline 106585882 & $c c 4$ & -3.5 & -3.2 & -9.5 & -8.5 & -4.7 & -2.0 & -9.3 & -2.8 \\
\hline 106577833 & $i l-8$ & nd & 1.6 & -3.2 & -1.3 & nd & 1.8 & 5.2 & 2 \\
\hline 106600843 & illifa/f1 & -11.3 & -12.2 & -10.8 & -9.6 & -2.5 & -3.2 & -10.9 & -8 \\
\hline 106611589 & lect2 & -3.1 & -2.3 & 36.8 & 45.9 & -3.7 & -2.2 & -6.9 & -5.8 \\
\hline 100136358 & nos 2 & -31.2 & -17.8 & -54.3 & -50 & -11.7 & -6.8 & -35.1 & -23.9 \\
\hline 106581433 & $p t x 3$ & nd & -1.2 & -1.4 & -1.7 & -1.4 & -2.1 & -2 & -2.5 \\
\hline 106604759 & sap & 2.2 & 2.5 & -1.4 & 1.1 & 1.7 & 2.9 & 1.6 & 2.5 \\
\hline \multicolumn{2}{|c|}{ Spearman correlation } & \multicolumn{2}{|c|}{$\rho=0.91, p<0.0002$} & \multicolumn{2}{|c|}{$\rho=0.89, p<0.0003$} & \multicolumn{2}{|c|}{$\rho=0.78, p<0.0008$} & \multicolumn{2}{|c|}{$\rho=0.91, p<0.0002$} \\
\hline
\end{tabular}

Table 1. Validation of RNA-seq data using qPCR. The expression of ten genes was analysed by qPCR across all experimental time points. Genes included acod1: aconitate decarboxylase 1, cath1: cathelicidin 1, clra: $c$ type lectin receptor a, cc4: c-c motif chemokine 4-like, lect2: leukocyte cell-derived chemotaxin 2-like, il-8:interleukin8-like, il17a/f1: interleukin-17a/f1-like, nos: nitric oxide synthase 2, inducible, ptx: pentraxin-related proteinlike, sap: serum amyloid P-component-like. nd: not detected, FC; fold change, dpi: days post infection. The RNA-seq and qPCR data were analysed using Spearman's rank order correlation. The correlation coefficient rho $(\rho)$ range is -1 to 0 to +1 , where 0 represents no correlation, -1 a negative correlation and +1 a positive correlation, $p$ is the statistical significance. Up-regulated genes are highlighted (bold).

motif chemokine 4-like, lect2: leukocyte cell-derived chemotaxin 2-like, il-8: interleukin-8-like, il-17a/f1: interleukin-17a/f1, nos2: nitric oxide synthase 2, inducible, ptx: pentraxin-related protein-like, sap: serum amyloid P-component-like. These genes were selected from the RNA-seq data for validation based on their expression at more than one time point and their involvement in the immune response (Table 1). The results of the qPCR confirmed that $a \operatorname{cod} 1, c c 4, i l-17 a / f 1$ and nos 2 , and $p t x 3$ were down-regulated at every time point. Cath1 was down-regulated at 4, 7 and $16 \mathrm{dpi}$. Two genes, clra and lect 2 showed the same pattern of expression being greatly up-regulated at $7 \mathrm{dpi}$, while being down-regulated at the other time points. Il-8 was down-regulated at $7 \mathrm{dpi}$ and up at $16 \mathrm{dpi}$. Sap was up-regulated at 4, 14 and again at $16 \mathrm{dpi}$ (Table 1). To use the qPCR data to validate the RNA-seq data, a Spearman correlation analysis was performed using the qPCR $\log _{2} \mathrm{FC}$ average of 6 individual fish for each of the ten genes. The correlation coefficient rho $(\rho)$ and statistical significance $(p)$ was 4 dpi: $\rho=0.91, p<0.0002,7$ dpi: $\rho=0.89, p<0.0003,14 \mathrm{dpi}: \rho=0.78, p<0.008$ and $16 \mathrm{dpi}: \rho=0.91, p<0.0002$.

Gene ontology (GO). GO and pathway enrichment analyses were undertaken to identify the function of genes whose expression was differentially expressed in the gill following exposure to $P$. perurans. DEGs mapped to 224 Gene Ontology (GO) terms of which 140 were categorised as a biological process (BP), 45 as cellular components (CC), and 39 as having a molecular function (MF). Multiple BP terms were identified for epithelial cell migration, RNA processing, positive regulation of protein catabolic process, canonical Wnt signaling, 
regulation of Type 1 interferon, and the ERK1/ERK2 cascade. The negative regulation of NF- $\kappa \beta$ (GO: 0032088) was also identified. Enriched ontology clusters were generated in Metscape ${ }^{24}$ at each time point $(4,7,14$ and $16 \mathrm{dpi}$ ) for terms associated with both down-regulated and up-regulated genes. In the top 20 ontology clusters enriched with down-regulated genes (Fig. 4), the reactone gene set R-DRE: 168256: Immune system was identified at $7 \mathrm{dpi}$ (Fig. 4B) while in the top 20 ontology clusters enriched with up-regulated genes, GO: 0002768: immune response-regulating cell surface receptor signalling pathway was identified at $4 \mathrm{dpi}$ (Fig. 5A) and $16 \mathrm{dpi}$ (Fig. 5D), with GO: 0002682: Regulation of immune system process identified at 14 dpi (Fig. 5C). The oxidation-reduction biological process (GO: 0055114, $-\log 10(P)$ 13.82) was identified as being the most enriched for up-regulated genes (108 genes) (Fig. 5B).

The top 20 down-regulated genes and the top 20 up-regulated genes at each for the 4 time points ( $4 \mathrm{dpi}, 7$ dpi, 14 dpi and 16 dpi) can be found in Supplementary Table S2 online and Supplementary Table S3 online, respectively.

Gene down-regulation. At 7 dpi pathway and process enrichment analysis identified R-DRE-168256: immune system as being enriched with 51 down-regulated genes (Fig. 4B, Supplementary Table S4 online) genes, while PPI also identified this reactome as being enriched with 77 down-regulated genes. Further analysis of these 77 identified genes with having involvement in KEGG signaling pathways including the NOD-like receptor (NLR) signaling (dre04621, FDR 5.68e-08 hsp90AB1, ikbkb, irf9, mapk3, nlrx1, sugt1, tbk1, stat1a, cybb), Toll-like receptor (TLR) signaling (dre04620, FDR 1.5e-04, tlr9, mapk3, tbk1, stat1a) and RIG-1-like receptor signaling (dre04622, FDR 3.2e-04, nlrx1, tbk1, mapk3k1, ikbkb). In addition to NOD, TLR and RIG-1 pattern recognition receptors (PRRs), other members of this family include C-type lectin-like receptors (CLR) and cytosolic DNA sensors ${ }^{25}$. PRRs respond to pathogen-associated molecular patterns (PAMPs) or host-derived damage-associated molecular patterns (DAMPs) by triggering activation of NF- $\kappa \beta$, AP1, CREB, c/EBP, and IRF transcription factors ${ }^{25}$. In the current study, the clra gene (GeneID 100136446) was substantially up-regulated at 7 dpi (Table 1), which would suggest that the NLR, TLR and RIG-1 pathway is regulated differently as the genes associated with these pathways are down-regulated at $7 \mathrm{dpi}$. The Herpes simplex infection pathway (dre05168: FDR 0.00015) was also identified as being enriched with down-regulated genes ( $i k b k b$, irf9, mapk3, stat1a, tap1, $t b k 1, t l r 9)$. The PPI also identified reactome gene sets enriched with down-regulated genes from R-DRE-168256: immune system, the most significant of these being R-DRE-1280215: Cytokine signalling in the immune system, containing 15 genes (FDR 2.8e-15, csf3r, dlg1, epgn, il-34, irf9, jak3, mapk3, nrg1, spred2b, stat4, syk, tbk1, tnfrsf11a, tnip2, ube2na) of which all the genes with the exception of 2 genes (tnfrsf11a and tnip2) were also associated with R-DRE-449147: Signalling by interleukins (Supplementary Table S4 online).

Cytokine gene expression. Interrogation of the DEG data set for interleukin ( $i l$ ) gene in AGD-affected gill identified multiple transcripts from nine $i l$ gene families whose expression was differentially expressed (Il$1 \beta, I l-8, i l-11, i l-12 \beta$, il-17 (a/d/f) il-18, il-27 $\beta$, il-3) (Table 2). Interestingly, il-17a/f1-like (Gene ID 106600843) was down-regulated at every time point. Two interleukins were found to be up-regulated,: $i l-27 \beta$ (Gene ID 106574502 ) was $12.4 \log _{2} \mathrm{FC}$ at $7 \mathrm{dpi}$, and one of the 2 paralogues of $i l-8$ (Gene ID 106577833) had a $2.4 \log _{2} \mathrm{FC}$ at $16 \mathrm{dpi}$.

The DEG lists were further screened for additional cytokines including interferons (ifn) (Supplementary Table S5 online), tumour necrosis factor-alpha $(\operatorname{tnf}-\alpha)$ and transforming growth factor- $\beta$ (tgf- $\beta$ ) (Supplementary Table S6 online). With regard to interferon, only one gene was found in the data set, ifna 3 which was downregulated at $16 \mathrm{dpi}\left(-1.3 \log _{2} \mathrm{FC}\right)$. Alpha/beta receptor la-like and gamma receptors (ifngr $\left.1 a, 1 b, 2 b\right)$ were found to be down regulated at the earlier time points of 4 and $7 \mathrm{dpi}$ while numerous interferon regulatory factors (irf) were down-regulated throughout the study (irf1, irf1 isoform 2, irf2a irf3, irf4-like, irf5, irf6-like, irf2a, irf7b, irf8like, irf9). irf2-like (GeneID 106612476) was up-regulated at $7 \mathrm{dpi}$ and $14 \mathrm{dpi}$, while two paralogues of irf4-like (GeneID 106599909, 106571913) were up-regulated at 14 dpi (Supplementary Table S5 online). Down-regulation of $t n f-\alpha\left(-3 \log _{2}\right.$ FC) was seen at 7 dpi with tnf- $\alpha 1$ precursor (Gene ID 100136457) persistently down-regulated from 4 to $14 \mathrm{dpi}\left(-1.4 \log _{2} \mathrm{FC} \pm 0.2\right)$ and the $t n f-\alpha 2$ precursor (Gene ID 100136458) down-regulated at the earlier times of $4 \mathrm{dpi}$ and $7 \mathrm{dpi}$. Upregulation of tgf- $\beta$ induced and tgf- $\beta 3$-like was evident at $7 \mathrm{dpi}$. One tgf- $\beta 2$-like (Gene ID 106584161) was up-regulated at both $7 \mathrm{dpi}$ and $16 \mathrm{dpi}$, while tgf- $\beta 1$-like (Gene ID 106569003) was up-regulated at both $14 \mathrm{dpi}$ and $16 \mathrm{dpi}$ (Supplementary Table S6 online).

Mucin gene expression. As AGD leads to the production of excessive mucus on the gills, the DEGs dataset was also interrogated for the expression of additional mucin gene expression (Supplementary Table S7 online). Mucins identified in the current study included muc-2-like, 5AC-like, 7-like, 12-like 13-like, 17-like and an intestinal mucin-like gene, with a possible similarity to $m u c$-2. Interestingly, there were 5 paralogues of muc-2-like identified in this study at the earlier times of $4 \mathrm{dpi}$ and $7 \mathrm{dpi}$, where all but one (Gene ID 106584523) was upregulated $\left(\log _{2} \mathrm{FC} 3.0\right)$. Two paralogues of $m u c$-5AC-like were present, with the expression of one (GeneID1 106612949) at 4 dpi up-regulated, while the other paralogue (GeneID1 106577588) was present at 7 dpi where the expression was down-regulated. One gene for muc-7-like was consistently down-regulated at every time point. The expression of muc-12-like was up-regulated $\left(\log _{2} \mathrm{FC} 0.9\right)$ at $14 \mathrm{dpi}$, while the expression of a muc-13like (GeneID 106579100) was substantially up-regulated $\left(\log _{2}\right.$ FC 7.8$)$ at $7 \mathrm{dpi}$.

Immune gene up-regulation. There were 14 immune-related genes up-regulated at times other than 7 dpi associated with various biological processes (Table 3), including GO: 0002682: Regulation of immune system process (btk, clcq, CD79b, CD99, cgas, hmgbla, hmgb3b, Il-34, kita, lyn, tfpi1, themis), GO: 0002253: Activation of immune system process (btk, c1cq, CD79b, cgas, hmgbla, themis), GO: 0097190: B-cell differentiation (cd79b, 
A

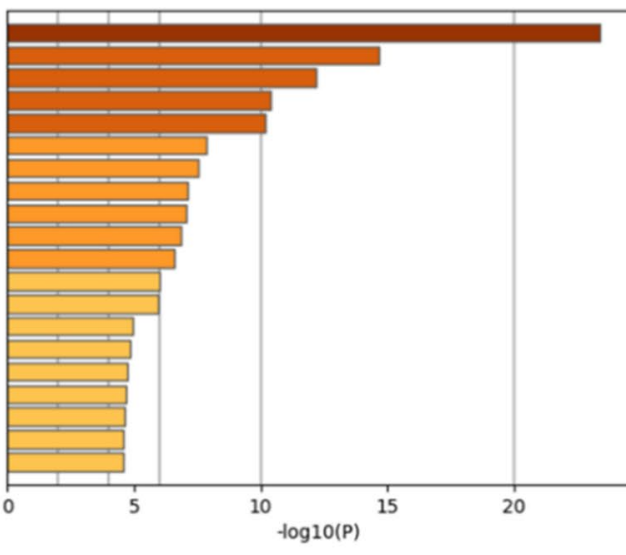

GO:0042254: ribosome biogenesis

GO:1990904: ribonucleoprotein complex GO:0030684: preribosome

GO:0006457: protein folding

dre03008: Ribosome biogenesis in eukaryotes

GO:0044429: mitochondrial part

GO:0044429: mitochondrial part

GO:1905368: peptidase

GO:0140098: catalytic activity, acting on RNA

R-DRE-5389840: Mitochondrial translation elongation

dre03030: DNA replication

GO:0043604: amide biosynthetic process

GO:0000154: rRNA modification

GO:0033260: nuclear DNA replication

GO:0005798: Golgi-associated vesicle

GO:0006403: RNA localization

dre00100: Steroid biosynthesis

GO:0030490: maturation of SSU-rRNA

dre04141: Protein processing in endoplasmic reticulum

GO:0004386: helicase activity

B

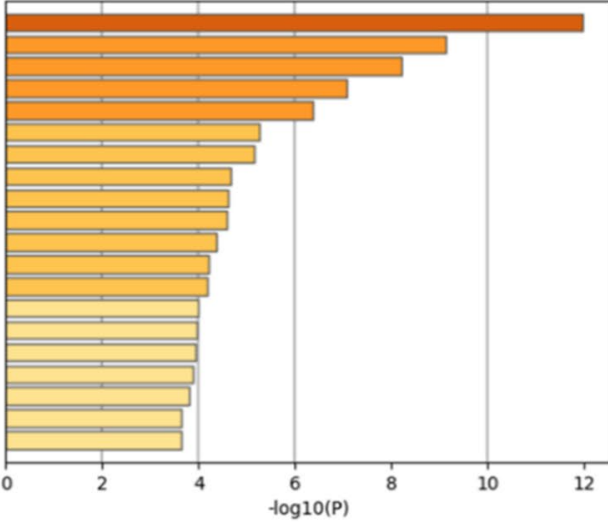

GO:0022402: cell cycle process

GO:0006260: DNA replication

R-DRE-194315: Signaling by Rho GTPases

dre04110: Cell cycle

GO:0010564: regulation of cell cycle process

dre03030: DNA replication

R-DRE-69481: G2/M Checkpoints

R-DRE-446210: Synthesis of UDP-N-acetyl-glucosamin

GO:0000779: condensed chromosome, centromeric region

R-DRE-168256: Immune System

GO:0043596: nuclear replication fork

GO:0005768: endosome

GO:0006396: RNA processing

GO:0090407: organophosphate biosynthetic process

GO:0008283: cell proliferation

R-DRE-74160: Gene expression (Transcription)

R-DRE-597592: Post-translational protein modification

GO:0015630: microtubule cytoskeleton

GO:0080134: regulation of response to stress

GO:0061564: axon development

C

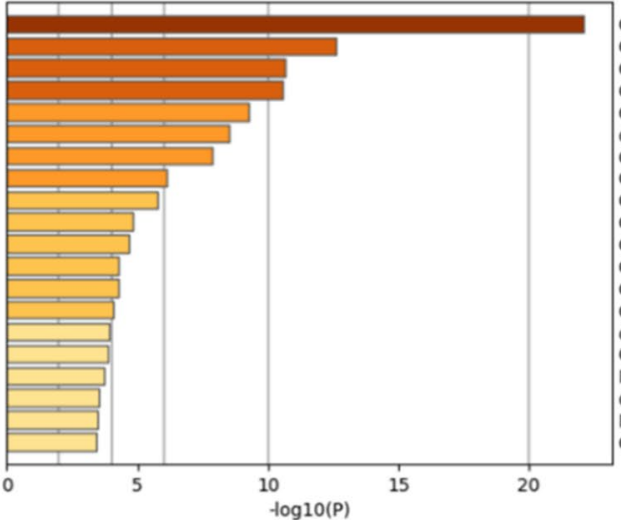

GO:0005730: nucleolus

GO:1990904: ribonucleoprotein complex

GO:0006261: DNA-dependent DNA replication

GO:0030684: preribosome

O:0140098: catalytic activity, acting on RNA

dre03008: Ribosome biogenesis in eukaryotes

60:0005657: replication fork

G:0016071: mRNA metabolic process

GO:0042273: ribosomal large subunit biogenesis

GO:0016874: ligase activity

GO:0031967: organelle envelope

O:0017056: structural constituent of nuclear pore

GO:0051082: unfolded protein binding

GO:0005694: chromosome

dre00240: Pyrimidine metabolism

GO:0000466: maturation of 5.8S rRNA from tricistronic rRNA transcript M00085: Fatty acid biosynthesis, elongation, mitochondria

dre03430: Mismatch repair.

M00341: Proteasome, 19S regulatory particle (PA700)

GO:0000278: mitotic cell cycle

$-\log 10(P)$

D

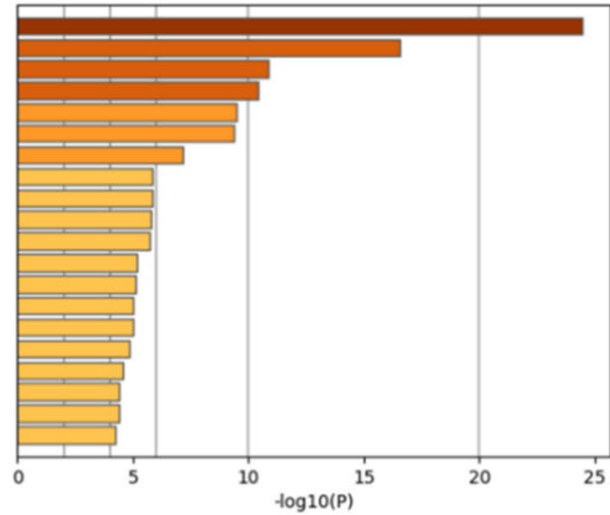

GO:0022613: ribonucleoprotein complex biogenesis GO:0140098: catalytic activity, acting on RNA

GO:0004386: helicase activity

GO:0030684: preribosome

dre03008: Ribosome biogenesis in eukaryotes

GO:1990904: ribonucleoprotein complex

G0:0006399: tRNA metabolic process

GO:0006260: DNA replication

GO:0006260: DNA replication

GO:0016874: ligase activity

GO:0016071: mRNA metabolic process

GO:0008757: S-adenosylmethionine-dependent methyltransferase activity

GO:0031967: organelle envelope

GO:0000727: double-strand break repair via break-induced replication

GO:0031123: RNA 3'-end processing

GO:0005643: nuclear pore

GO:0019752: carboxylic acid metabolic process

dre00240: Pyrimidine metabolism

dre00100: Steroid biosynthesis

M00341. Proteasome, 19S regulatory particle (PA700)

$\log 10(P)$

Figure 4. Top 20 ontology clusters enriched with down-regulated genes. (A) 4 dpi, (B) 7 dpi, (C) 14 dpi and (D) $16 \mathrm{dpi}$. The $\mathrm{x}$-axis is the $p$-value in negative log base 10. The darker the colour of the horizontal column, the more significant the cluster enrichment. Danio rerio (dre) was used as the fish model species in Metscape $^{24}$, where the naming convention for KEGG pathway is dre, and the reactome gene set is R-DRE. 


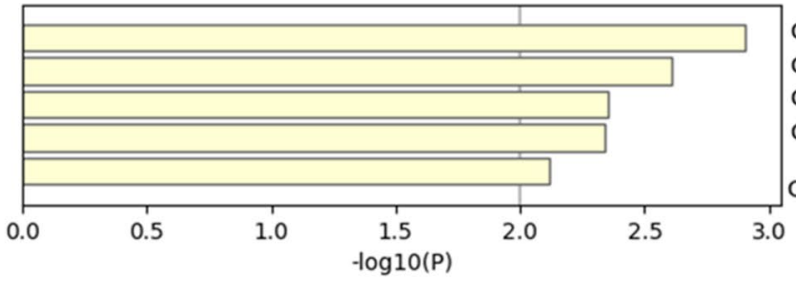

GO:0005096: GTPase activator activity

GO:0008643: carbohydrate transport

GO:0000323: lytic vacuole

GO:0002768: immune response-regulating GO:0048878: chemical homeostasis

B

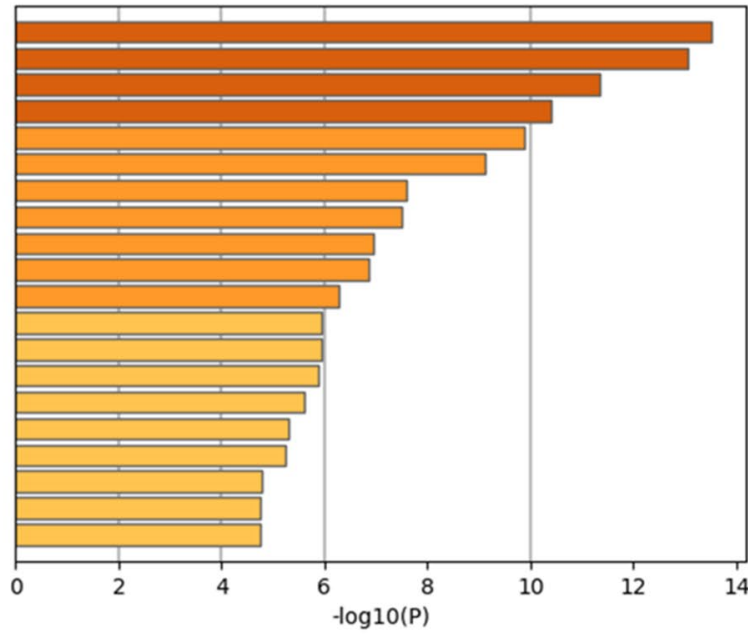

GO:0055114: oxidation-reduction process

GO:0019752: carboxylic acid metabolic process

GO:0032787: monocarboxylic acid metabolic process

GO:0006629: lipid metabolic process

dre04146: Peroxisome

R-DRE-1430728: Metabolism

GO:0044283: small molecule biosynthetic process

dre03010: Ribosome

dre00380: Tryptophan metabolism

dre00260: Glycine, serine and threonine metabolism

dre04142: Lysosome

dre00340: Histidine metabolism

GO:0051186: cofactor metabolic process

GO:0005783: endoplasmic reticulum

GO:1901565: organonitrogen compound catabolic process

GO:0044429: mitochondrial part

GO:0017144: drug metabolic process

GO:0005975: carbohydrate metabolic process

dre01040: Biosynthesis of unsaturated fatty acids

GO:0009074: aromatic amino acid family catabolic process

\section{C}

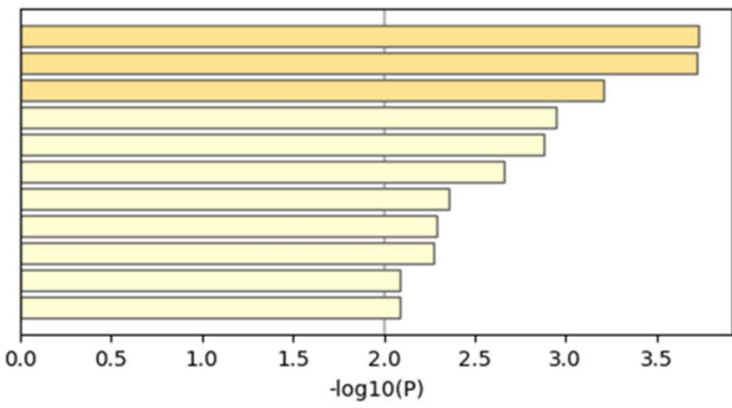

GO:0001614: purinergic nucleotide receptor activity GO:0002682: regulation of immune system process GO:0038083: peptidyl-tyrosine autophosphorylation GO:0042537: benzene-containing compound metabolic process GO:0065009: regulation of molecular function GO:0008301: DNA binding, bending GO:0097190: apoptotic signaling pathway GO:0008238: exopeptidase activity GO:0046906: tetrapyrrole binding GO:0003171: atrioventricular valve development GO:0008643: carbohydrate transport

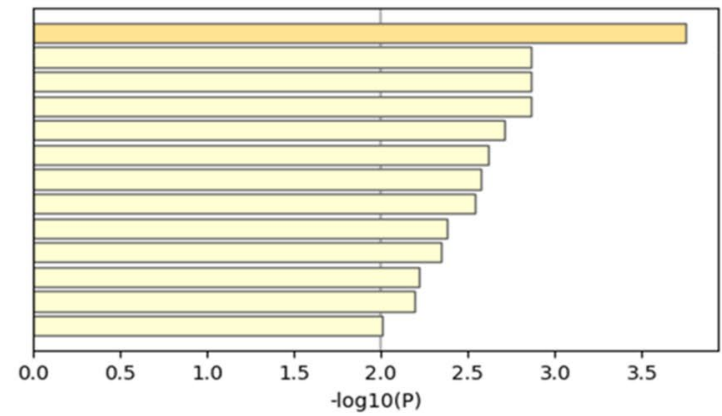

GO:0065009: regulation of molecular function GO:0014013: regulation of gliogenesis GO:0070374: positive regulation of ERK1 and ERK2 cascade GO:0042537: benzene-containing compound metabolic process GO:0002768: immune response-regulating cell surface receptor signaling pathway GO:0030234: enzyme regulator activity R-DRE-1433557: Signaling by SCF-KIT GO:0032989: cellular component morphogenesis GO:0000045: autophagosome assembly GO:0008376: acetylgalactosaminyltransferase activity GO:0004725: protein tyrosine phosphatase activity GO:0008509: anion transmembrane transporter activity GO:0008643: carbohydrate transport

Figure 5. Ontology clusters enriched with up-regulated genes. (A) 4 dpi: 5 clusters, (B) 7 dpi: $>20$ clusters, (C) 14 dpi: 11 clusters and (D) 16 dpi: 13 clusters. The x-axis is the $p$-value in negative log base 10. The darker the colour of the horizontal column, the more significant the cluster enrichment. Danio rerio (dre) was used as the fish model species in Metscape ${ }^{24}$, where the naming convention for KEGG pathway is dre, and the reactome gene set is R-DRE.

Ikzf1.7, kita), GO: 0002768: immune response-regulating cell surface receptor signalling pathway (cd79b, kita, themis2). Genes were also associated with the Reactome gene set R-DRE-983695: Antigen activates B-cell receptor (BCR) leading to generation of second messengers ( $b t k, c d 79 b$, dapp). Immune activation was evident from 7 dpi with complement activation (DRE-166658, FDR 0.0178) involving ten genes (crp, $c 6, c 8 a, c 8 b, c 8 g, c 9, c p b 2$, $h b l 3$, masp1, masp2) and is consistent with what was found in the proteome analysis of AGD-affected gill in our laboratory ${ }^{26}$. The most up-regulated gene was mannan binding lectin serine peptidase $2\left(\operatorname{masp} 2,30.0 \log _{2} \mathrm{FC}\right)$ gene ID 106572058). Screening of the top 100 genes identified thirteen other complement transcripts also up- 


\begin{tabular}{|c|c|c|c|c|c|}
\hline Time & GeneID & Description & Chr & p-adj & $\log _{2} \mathrm{FC}$ \\
\hline \multirow{5}{*}{$4 \mathrm{dpi}$} & 106570815 & il-1 beta-like & ssa15 & 0.0008 & -1.6 \\
\hline & 100136449 & il-1 beta & ssa24 & 0.0221 & -0.9 \\
\hline & 106601367 & il-17A-like & ssa01 & 0.0016 & -1.1 \\
\hline & 106600843 & il-17A/F1-like & ssa01 & 0.0000 & -3.5 \\
\hline & 106571057 & il-17F-like & ssa15 & 0.0000 & -2.5 \\
\hline \multirow{14}{*}{$7 \mathrm{dpi}$} & 106570815 & il-1 beta-like & ssa15 & 0.0005 & -2.6 \\
\hline & 106562915 & il-1 beta-like & ssa11 & 0.0235 & -1.2 \\
\hline & 100136449 & il-1 beta & ssa24 & 0.0014 & -2 \\
\hline & 100195681 & $i l-8$ & ssa06 & 0.0037 & -1.7 \\
\hline & 106575827 & il-11 & ssa02 & 0.0061 & -2.3 \\
\hline & 106603888 & il-12 subunit beta-like & ssa04 & 0.0098 & -1.9 \\
\hline & 106612126 & il-12 subunit beta-like & ssa09 & 0.0203 & -1.6 \\
\hline & 101448031 & il-15 & ssa04 & 0.0069 & -1.2 \\
\hline & 106600843 & il-17A/F1-like & ssa01 & 0.0000 & -3.4 \\
\hline & 106571057 & il-17F-like & ssa15 & 0.0010 & -2.4 \\
\hline & 100196379 & Il-18 & ssa20 & 0.0041 & -1.4 \\
\hline & 106574502 & il-27 subunit beta-like & ssa16 & 0.0000 & 12.4 \\
\hline & 106560860 & il-34 & ssa10 & 0.0426 & -0.7 \\
\hline & 106573710 & il-34-like & ssa16 & 0.0402 & -1.3 \\
\hline $14 \mathrm{dpi}$ & 106600843 & il-17A/F1-like & ssa01 & 0.0053 & -1.3 \\
\hline \multirow{9}{*}{$16 \mathrm{dpi}$} & 100136449 & il-1 beta & ssa24 & 0.0075 & -0.9 \\
\hline & 106562915 & il-1 beta-like & ssal1 & 0.0103 & -0.8 \\
\hline & 106570815 & il-1 beta-like & ssa15 & 0.0009 & -1.4 \\
\hline & 106577833 & il-8-like & ssa18 & 0.0210 & 2.4 \\
\hline & 100195681 & Il-8 & ssa06 & 0.0039 & -1 \\
\hline & 106612126 & il-12 subunit beta-like & ssa09 & 0.0069 & -0.6 \\
\hline & 100195864 & $I l-17 D$ & ssa21 & 0.0202 & 0.6 \\
\hline & 106571057 & il-17F-like & ssa15 & 0.0184 & -0.8 \\
\hline & 106600843 & il-17A/F1-like & ssa01 & 0.0000 & -3.4 \\
\hline
\end{tabular}

Table 2. Relative gene expression of Interleukins in AGD-affected gill. GeneID: Salmo salar gene identification number, chr: chromosome location, p-adj: $P$-values adjusted for false discovery rate, $\log _{2} \mathrm{FC}: \log$ of gene expression fold change to base 2, up-regulated genes are highlighted with bold text, dpi: days post inoculation.

regulated including (C1q-like protein $2(\times 4)$, C1q-like protein 3, C2-like, C3-like $(\times 2)$, C9, factor B, factor H, properdin-like $(\times 2)$. Also up-regulated at $7 \mathrm{dpi}$ were genes involved in the acute phase response, an early innate immune function that is initiated by inflammatory signals, leading to the release of acute phase proteins into the bloodstream to re-establish homeostasis following microbial infection ${ }^{27}$. Genes involved in the acute phase response (APR) markedly up-regulated at $7 \mathrm{dpi}$ in the AGD affected gill included pentraxin, alpha-1-antitrypsin, alpha-2-macroglobulin, fibrinogen $(\alpha, \beta, \gamma)$, leukocyte cell-derived chemotaxin-2 (lect2), C-type lectin receptor (clra), and serum amyloid P-component (sap) (data not shown).

\section{Discussion}

Despite the growing number of gene expression and proteomic studies being carried out on AGD-affected salmon (reviewed in Marcos-Lopez \& Rodger 2020) ${ }^{4}$, there are still knowledge gaps remaining regarding host immune response in early stage disease. The progression of AGD on the gill can be monitored visually and scored based on the absence or presence of mucoid patches and their distribution on the gills ${ }^{6}$. In the current study, AGD mucoid lesions were not evident on the gills in AGD-affected groups at the earlier time points (4 and $7 \mathrm{dpi}$ ) and the gill score in the AGD-affected fish remained less than 2 by day 16 . Although it is known that long term cultivation of $P$. perurans results in the loss of parasitic virulence in $v i v 0^{28}$, the amoeba used in the current study were twelve weeks in culture prior to use in the challenge trial. The progression of the disease based on gill scores observed raised a concern in relation to the virulence of the pathogen. However, at the first sampling point (4 dpi) P. perurans DNA was detected by qPCR and microscopically, there was evidence of epithelial hyperplasia. Amoebic virulence was therefore shown to be adequate and the rate of disease progression appropriate in order to investigate gene expression at early disease stages. Moreover, at $4 \mathrm{dpi}, 4838$ genes were differentially regulated with 2985 genes down-regulated and 1853 up-regulated. By 7 dpi the pattern of gene expression changed, with more genes up-regulated and to a greater extent (Fig. 2). In addition to hyperplasia, fusion of the lamellae was now evident, indicative of AGD in the absence of visible mucoid patches. At $14 \mathrm{dpi}$ and $16 \mathrm{dpi}$, the pattern of gene expression returned to that which was seen at 4 dpi. Principal component analysis of the 7 dpi time point showed four of the six AGD-affected samples clustered together and were clearly different from the other fish 


\begin{tabular}{|l|l|l|l|}
\hline Gene ID & Gene symbol & Biological process and pathway & dpi \\
\hline 568653 & $b t k$ & GO: 0002682, GO: 0002253, R-DRE-983695 & 14,16 \\
\hline 449803 & $c l q c$ & GO: 0002682, GO: 0002253 & 14,16 \\
\hline 100329481 & $c d 79 b$ & $\begin{array}{l}\text { GO: } 0002682, \text { GO: 0002253 } \\
\text { GO: } 0097190, \text { GO: } 0002768, \text { R-DRE-983695 }\end{array}$ & $4,14,16$ \\
\hline 559896 & $c d 99$ & GO: 0002682 & 14 \\
\hline 557043 & $c g a s$ & GO: 0002682, GO: 0002253 & 14,16 \\
\hline 550386 & dapp1 & R-DRE-983695 & 14,16 \\
\hline 321622 & hmgbla & $\begin{array}{l}\text { GO: } 0002682 \\
\text { GO: } 0002253\end{array}$ & 14,16 \\
\hline 550466 & hmgb3b & GO: 0002682 & 14 \\
\hline 560193 & Il-34 & GO: 0002682 & 14 \\
\hline 30256 & kita & $\begin{array}{l}\text { GO: } 0002682 \\
\text { GO: } 0097190 \\
\text { GO: } 0002768\end{array}$ & $4,14,16$ \\
\hline 30177 & ikzf1 & GO: 0097190 & 14,16 \\
\hline 447804 & lyn & GO: 0002682 & 14 \\
\hline 560339 & tfpi2 & GO: 0002682 & 14 \\
\hline 100535600 & themis2 & $\begin{array}{l}\text { GO: } 0002682 \\
\text { GO: } 0002253\end{array}$ & $4,14,16$ \\
\hline
\end{tabular}

Table 3. Genes up-regulated in immune regulation and activation at 4, 14 and $16 \mathrm{dpi}$, but not at $7 \mathrm{dpi}$, in AGD-affected gill. Gene symbols: btk: bruton agammaglobulinemia tyrosine kinase, clqc: complement component 1, q subcomponent, C chain, cd79b: cd79b molecule, immunoglobulin-associated beta, cgas: cyclic GMP-AMP synthase, hmgb: high mobility group box, il-34: interleukin-34, kita: kit proto-oncogene, receptor tyrosine kinase a, ikzf1:IKAROS family zinc finger 1, lyn:lyn proto-oncogene, Src family tyrosine kinase, tfpi2: tissue factor pathway inhibitor 2, themis2: thymocyte selection associated family member 2. Biological processes: GO: 0002682: Regulation of immune system process, GO: 0002253: Activation of immune system process, GO: 0097190: B-cell differentiation, GO: 0002768: immune response-regulating cell surface receptor signalling pathway. Reactome gene set: R-DRE-983695: Antigen activates B-cell receptor (BCR) leading to generation of second messengers. dpi: days post inoculation.

sampled. With the other samples in the $7 \mathrm{dpi}$ AGD-affected group, one sample clustered with the pre-7 dpi samples, while the other sample clustered with the post-7 dpi samples. The fish were exposed to the P. perurans by immersion. In comparison to intraperitoneal injection methods, the number of amoeba colonising the gill of each fish cannot be controlled, so this finding was not unexpected and highlights the importance of using individual instead of pooled samples for fish studies, and having a minimum of 6 fish per group for RNA-seq AGD studies ${ }^{29}$. For this study, the same gill (gill 2) was taken from each fish with no pre-selection for the presence or absence of mucoid lesions.

The RNA-seq data was validated using qPCR analysis of a panel of immune genes. Down regulated genes at each time point included $a \operatorname{cod} 1, c c 4, i l-17 a / f 1$ and nos 2 , and $p t x 3$ with cath 1 being down-regulated at 4,7 and 16 dpi. Acod1, also known as immune responsive gene 1 (irgl) is a gene coding for an enzyme producing itaconic acid through the decarboxylation of cis-aconitate. The expression levels of acod1/irg1 correlates to itaconic acid production, an indicator of inflammation activation in mammals ${ }^{30}$. The $\operatorname{acod} 1$ gene is emerging as a regulator of immuno-metabolism in inflammation and infection ${ }^{31}$. In the current study, the pattern of expression of acod1/irg1 and nos 2 were similar with both genes being consistently down-regulated. Furthermore, both nos 2 and acod 1 genes were also shown to have involvement in the biological process 'GO: 0072593: Reactive oxygen species metabolic process' containing 7 down-regulated genes (nos 1, ncfl, cybb, nos $2 a$, noxa 1, hace 1, acod1) at 7 dpi. The lack of an increase in nos 2 expression in the current study, is consistent with the previous finding that there was no detectable increase in the expression of iNos (nos2) between healthy and AGD-affected fish during early disease stage ${ }^{15}$. In a previous study, the differential expression of the superoxide dismutase (sod) and catalase (cat), two genes involved in oxidative stress, were reported to be up-regulated in late stage AGD ${ }^{20}$. SOD catalyses the dismutation of the superoxide $\left(\mathrm{O}_{2}^{-}\right)$into $\mathrm{O}_{2}$ or $\mathrm{H}_{2} \mathrm{O}_{2}$ while CAT plays a role in protecting cells from oxidative damage. In the current study, at 7 dpi there were 108 genes identified as being up-regulated in GO: 0055114, the oxidation-reduction biological process (Fig. 5B). Three sod genes were found to be up-regulated including soluble sod1, (GeneID: 30553), the mitochondrial sod2 (GeneID: 335799) and the extracellular sod3b (GeneID: 794006). In the same study, cat gene expression was reported as being significantly upregulated in gill lesion tissue with gill score 2. In the current study, catalase was not found to be up-regulated prior to the appearance of lesions on the gill, at $7 \mathrm{dpi}$, further confirming this gene as a possible lesion specific marker for AGD. The up-regulation of genes involved in the oxidative-reduction biological process during early stage AGD will be further investigated in a future study. TGF- $\beta 1$ is a potent suppressor of nos 2 by multiple mechanisms in numerous cell types, including macrophages ${ }^{32}$. An up-regulation of $\operatorname{tg} f-\beta$ isoforms $(\beta 1, \beta 2$, induced) was evident in the current study from $7 \mathrm{dpi}$. TGF- $\beta$ can promote IL-17 cell differentiation however the expression of $I l-17 a /$ $f 1$ was consistently down-regulated. CC4 is a chemokine for natural killer cells, monocytes and a variety of other 
immune cells ${ }^{33}$. LECT2 is a chemotactic factor attracting neutrophils to the site of infection. The genes lect2 and clra showed the same pattern of expression being substantially up-regulated at $7 \mathrm{dpi}$, while being down-regulated at the other time points. Indeed it is the interaction between LECT2 and C-type lectin receptor (CLRA) proteins that is thought to be responsible for the "neutrophil-chemotactic" characteristic of LECT2 ${ }^{34}$. Furthermore, Lect2, along with $p t x$ and sap are acute phase response genes. Interestingly, the expressions of $p t x$ and sap were not as expected. While ptx (GeneID 106581433) was found to be down regulated, and sap (GeneID 106604759) upregulated approximately twofold, paralogues of sap (GeneID 106608633) and ptx (GeneID 100136583) were both substantially up-regulated at $7 \mathrm{dpi}$. Previous findings reported no differential gene expression of an SAP pentraxin in AGD-affected gill having visible mucoid lesions ${ }^{13}$. It is noteworthy that $\mathrm{Il}-8$, also a chemotactic factor with similar functions to lect2, did not show a similar expression trend, and was down-regulated at $7 \mathrm{dpi}$, and up-regulated at $16 \mathrm{dpi}$.

From the RNA-seq data set, down-regulated immune genes at 7 dpi were identified in the Reactome gene set R-DRE-68256: Immune system, which were associated with NOD-like receptor (NLR), Toll-like receptor (TLR) and RIG-1 like receptor signaling pathways, and also cytokine and interleukin signalling. NOD-like receptors (NLRs) can initiate or regulate host defence pathways through formation of signalling platforms that subsequently trigger the activation of inflammatory caspases and NF- $\kappa \beta^{35}$. A recent study of advanced stage AGD (day 21, gill score 3.3) reported activation of such defence pathways, with upregulation of genes mapped to NLR, TLR signalling, and Herpes simplex virus 1 infection pathways ${ }^{36}$. The current study also identified NLR and TLR signalling pathways, but enriched for down-regulated genes at 7 dpi. Involvement of the Herpes simplex virus 1 infection pathway suggests a viral-like response to parasite invasion in $\mathrm{AGD}^{36}$. In the current study the Herpes simplex infection pathway (dre05168: FDR 0.00015) was also enriched with down-regulated genes at 7 dpi, suggesting that the anti-viral response is either not activated, or suppressed, in early stage AGD, a theory that is further supported by down-regulation of genes associated with the biological process GO: 0060337: Type 1 interferon signaling pathway, and interferon regulatory factors. Other pathways enriched with down-regulated genes at $7 \mathrm{dpi}$ included B-cell receptor (BCR) signalling, T-cell receptor (TCR) signalling, the differentiation of Th1/Th2 cells, and Th17 cells. Interestingly, these signalling pathways had 2 genes in common, ikbkb and mapk3. Host invasion by pathogens frequently induces activation of NF- $\kappa \beta$, which plays an important role in initiation of innate immune responses by regulating the expression of many immunological mediators, including chemokines, cytokines, adhesion molecules, and enzymes that produce secondary inflammatory mediators ${ }^{37}$. Based on the pattern of gene expression in the current study, specifically at $7 \mathrm{dpi}$, P. perurans may have developed an immune evasion strategy to prevent the activation of NF- $\kappa \beta$ during the early onset of AGD. Of particular interest is the down regulation of genes involved in NOD-like receptor pathway, perpetuating the inhibition of NF- $\kappa \beta$ by Iк $\beta$. Pathogens have previously been reported to have developed strategies to circumvent the activation of the NF- $\kappa \beta$ activation, by preventing the inhibitor, I $\kappa$, from being ubiquitinated and therefore preventing its degradation, causing NF- $\kappa \beta$ to remain sequestered in the cell cytoplasm and therefore inactive ${ }^{38}$. Indeed, some viruses for example vaccinia viral protein $\mathrm{B} 14$, encode virulence factors, to target IKK $\beta$ to inhibit the NF- $\kappa \beta$-mediated antiviral immune response ${ }^{39}$. Therefore it is conceivable that $P$. perurans virulence factors could potentially have some immunomodulatory effects on their host.

With regard to the down-regulation of genes with involvement in cytokine and interleukin signalling, interrogation of the DEG dataset identified multiple transcripts from nine interleukin ( $i l$ ) gene families with differential expression in AGD-affected gills, with one gene il-17a/f1-like (Gene ID 106600843) was found to be down-regulated at every time point. Only two interleukins were found to be substantially up-regulated, il-27 $\beta$ at 7 dpi, (12.4 $\log _{2}$ FC), and one of 2 paralogues of $i l-8$ (Gene ID 106577833) at $16 \mathrm{dpi}\left(2.4 \log _{2} \mathrm{FC}\right.$ ). The up-regulation of $I l-1 \beta$ has been reported as the hallmark of late stage AGD infection ${ }^{17}$ associated with larger AGD-lesions. In these cases the observed increase in mucous cell hyperplasia has led to the contention that mucous cells are the potential source of $i l-1 \beta^{13}$. Multiple paralogues of $i l-1 \beta$ were identified in the current study with down-regulated expression seen at $4 \mathrm{dpi}, 7 \mathrm{dpi}$ and $16 \mathrm{dpi}$, suggestive of an impaired inflammatory response. Two paralogues of Il-12 $\beta$-like, the p40 subunit for IL-12 and IL-23 were also down-regulated at $7 \mathrm{dpi}$. IL-12 is a growth factor for Th $1^{40}$ while IL-23 is involved in Th17 cell differentiation ${ }^{41}$. IL-17 is a key cytokine produced by Th17 cells, involved in the inflammatory and neutrophil response. IL-17A is produced mainly in T cells, whereas IL-17F is produced in T cells, innate immune cells, and epithelial cells ${ }^{42}$. The role of IL-17F is mainly in the mucosal host defence mechanisms ${ }^{43}$. A recent study reported the expression of $I l-17 a / f 1 b$ and $I l-17 d$ to be significantly down-regulated in comparison to the negative control in gills from fish inoculated with a high concentration of P. perurans trophozoites $(5000 \text { amoeba } / \mathrm{L})^{12}$. In the current study where fish were infected with 2750 amoeba/L, Il-17a-like was also found to be down-regulated at $4 \mathrm{dpi}$, while $i l-17 a / f 1$ was consistently down-regulated at every time point. Interleukins involved in inducing IFN- $\gamma$, which promotes Th1 cell differentiation, $I l-15$ and $I l-18$, were also down-regulated in the current study. Interestingly, the expression of $\mathrm{Il}-27 \beta$, a component of both interleukins IL-27 and IL-35, was substantially up-regulated at $7 \mathrm{dpi}\left(12.4 \log _{2}\right.$ FC). IL-27 is unique in that although it induces Th1 differentiation, it can antagonise the development of the Th17-cell response and limit Th-17 driven inflammation ${ }^{44}$, critical for host defence against bacterial, fungal and viral infections at mucosal surfaces ${ }^{40}$ while IL-35 induces proliferation of Treg cell populations but reduces activity of Th17 cell populations ${ }^{45}$. In addition to the down-regulation of interleukins, numerous ifn receptors and regulatory factors were down-regulated throughout the study, with the exception of irf2-like and irf4-like which were both up-regulated at $14 \mathrm{dpi}$. The down-regulation of $\operatorname{tnf}-\alpha$ was also evident.

Mucins are high molecular weight glycoproteins secreted by goblet cells and the main structural component of mucus. Excessive mucus production in the gills is a hallmark of $\mathrm{AGD}^{3}$ with substantial up-regulation of the secreted MUC5AC detected in clinical AGD ${ }^{14}$. Specialized epithelial (goblet) cells are the major source of MUC5AC, which can be induced by MMP9 through the activation of the epidermal growth factor receptor (EGFR) and mitogen-activated protein kinase 3/2 MAPK 3/2(ERK1/2) cascade ${ }^{46}$. The expression of three genes 
(kita, hmgb1a, gpr183a) involved in the positive regulation of the ERK1/2 cascade (GO: 0070374) were found to be upregulated at the last time point (16 dpi) in this study. Mucins (muc) identified in the current study included muc-2-like, 5AC-like, 7-like, 12-like 13-like, 17-like and an intestinal mucin-like gene (Supplementary Table S7 online). Two paralogues of $m u c$-5AC-like were found to be differentially expressed, with one paralogue at $4 \mathrm{dpi}$ up-regulated, while the other paralogue at $7 \mathrm{dpi}$ was down-regulated. Interestingly, the expression of a muc-13-like was found to be substantially up-regulated $\left(\log _{2} \mathrm{FC} 7.8\right)$ at $7 \mathrm{dpi}$. Muc-13 is associated with mucosal immunity, and is an epithelial and hemopoietic cell surface mucin that protects against inflammation and may also play a role in cell signaling ${ }^{47,48}$. Muc-13 has also been reported as a quantifiable host marker of plasmodium parasite infection which could potentially be used to distinguish infected from uninfected cells ${ }^{49}$. A recent RNAseq study also reported the differential expression of a mucin-13-like gene in the head-kidney of advanced stage AGD-affected fish but not the gill ${ }^{36}$. Further investigation is warranted to determine the role of muc-13-like in the various tissues of AGD-affected fish and if the expression of this mucin is triggered through P. perurans cell attachment.

The present study provides the initial discovery and description of cytokine down-regulation during the amoeba attachment phase of early-stage AGD in the gill of Atlantic salmon, and provides the basis for future, more in-depth studies to elucidate the mechanisms behind this potential immune evasion strategy.

\section{Methods}

Study design. This early stage AGD study was designed to compare the transcriptomic profile from individual gill tissue from 6 fish at 4 time points $(4,7,14$ and $16 \mathrm{dpi})$ to 6 uninfected control fish $(0 \mathrm{dpi})$. As the 0 dpi time point was critical, it was decided to include two groups of 6 control fish (T0_GCRL, T0_GAGD) in the RNA sequencing. As all six fish in both control groups had a similar gene profile, the T0_GCRL group was subsequently selected for the data analysis. The experiment was designed to establish an AGD challenge with a type I error of $5 \%$ assuming a success rate of $80 \%$ (power analysis) and also taking into consideration a recent paper on biological replicates for RNA-seq ${ }^{29}$.

Fish husbandry. Atlantic salmon smolts $(78.8 \pm 20.6 \mathrm{~g})$ were sourced from a local freshwater hatchery on the west coast of Ireland and transported to the Marine and Freshwater Research Center, at the Galway Mayo Institute of Technology. Fish are were distributed into 8 circular black $1 \mathrm{~m}^{-3} \mathrm{~L}$ tanks (45 fish/tank) connected to recirculating aquaculture systems at a stocking density of $3.6 \mathrm{~kg} \mathrm{~m}^{-3}$, water temperature $12{ }^{\circ} \mathrm{C}$, artificial seawater (30ppt) and a $12 \mathrm{~h} / 12 \mathrm{~h}$ light/dark cycle. Fish were acclimatized for 6 days prior to the start of the trial. Fish were fed a commercial salmon diet (Le Gouessant) at $1 \%$ body weight per day. The in-vivo fish trial was carried out according to the ARRIVE guidelines for animal research ${ }^{50}$. This project was authorised by the Health Products Regulatory Authority (HPRA), authorisation number AE19137/P001, in compliance with Directive 2010/ 63/ EU transposed into Irish law by S.I. No 543 of 2012.

Paramoeba perurans isolation and culture. Paramoeba perurans trophozoites were collected by gill swabbing from AGD infected Atlantic salmon on a commercial salmon farm on the west coast of Ireland. Amoebae were cultured on marine yeast agar plates (MYA; $0.01 \%$ malt, $0.01 \%$ yeast, $2 \%$ Bacto Agar), $16{ }^{\circ} \mathrm{C}$ overlaid with $7 \mathrm{ml}$ sterile sea water ${ }^{51}$, and sub-cultured weekly by transferring free-floating cells to fresh MYA plates. Confirmation of $P$. perurans identity was performed using qPCR as previously described by Downes $(2015)^{5}$. The amoeba was in culture for 12 weeks prior to challenge.

Paramoeba perurans challenge. Six MYA agar plates were scraped gently and the sterile seawater (SSW) overlay and cells collected into a $2 \times 50 \mathrm{ml}$ tubes. The volume of each tube was brought up to $40 \mathrm{ml}$ using SSW at $16^{\circ} \mathrm{C}$, centrifuged at $800 \times g$ for $10 \mathrm{~min}$ at $16^{\circ} \mathrm{C}$. The supernatant, with the exception of the last $5 \mathrm{ml}$ was removed from each tube. The pellet was re-suspended in the remaining $5 \mathrm{ml}$ and then both $5 \mathrm{ml}$ were combined into one $10 \mathrm{ml}$ concentrate of amoeba. In a $15 \mathrm{ml}$ tube, a 1:40 dilution was made using $250 \mu \mathrm{l}$ of amoeba into $9.75 \mathrm{ml} \mathrm{SSW}$. The tube was closed and inverted several time to ensure proper distribution of the amoeba. The diluted amoeba $(1 \mathrm{ml})$ were placed on a Sedgewick rafter counter chamber. The cells were allowed to settle on the chamber for 10 min prior to counting. Counts were performed in triplicate. After an acclimatisation period of 6 days, 90 fish were challenged with $P$. perurans $(2750$ amoebae/L) in $300 \mathrm{~L}$ for $4 \mathrm{~h}$ in artificial seawater (30ppt), with oxygen saturation, water temperature $\left(12^{\circ} \mathrm{C}\right)$, and fish behaviour and welfare closely monitored. The 90 control fish were also held at $300 \mathrm{~L}$ for $4 \mathrm{~h}$ under the same conditions without $P$. perurans. Following the challenge, fish were returned to their respective tanks to be held under the same conditions as prior to challenge.

Disease progression. Clinical symptoms of AGD were determined by macroscopic examination of the intact gills in euthanised fish and were scored according to Taylor et al. ${ }^{6}$. Prior to sampling, 3 fish were taken from each duplicate control and AGD-affected tanks, and all 6 fish placed in an anaesthetic bath. Naïve control fish (6) and experimental fish (6) were sampled at each time point (0, 4, 7, 14 and 16 dpi) and all fish were gill scored.

Sample collection. The current study was carried out in parallel with another study investigating the proteomic expression in early stage AGD from fish (6 control, 6 AGD-treated) sampled at 9 time points over 16 days $(0,1,2,3,4,7,9,11,14,16 \mathrm{dpi})^{26}$. Prior to sampling, 3 fish were taken from each duplicate control and AGD-affected tanks, and all 6 fish placed in anaesthetic at the same time. Fish were euthanised by overdose of anaesthetic (400 $\mathrm{mg} \mathrm{L}^{-1}$ tricaine methane sulfonate) and the gills examined and scored based on the absence (0) 
or presence ( 1 to 5 ) of the mucoid patches on euthanised fish ${ }^{6}$. Prior to the tissue sampling, the fish were bled from the caudal vein. To minimize blood contamination in gill tissue, prior to extraction, the gills were perfused by injection of $2 \mathrm{ml}$ phosphate buffered saline (PBS) into the heart. For RNA sequencing the entire second left gill, irrespective of the presence of absence of mucoid lesions, was removed with the arch and placed in $300 \mathrm{ul}$ of RNAlater (Ambion Inc, Austin, Texas), stored overnight at $4{ }^{\circ} \mathrm{C}$ before being transferred to $-80^{\circ} \mathrm{C}$. For histopathology, the first right gill with arch was fixed in $10 \%$ buffered neutral formalin solution prior to being routinely processed and embedded in paraffin wax blocks. Gill tissue sections $(5 \mu \mathrm{m})$ were stained with haematoxylin and eosin (H\&E), and examined using an Olympus BX41 Microscope and CellSens software (Olympus, Tokyo, Japan) and imaged.

For amoeba identification, the fourth right gill with the arch was removed from individual fish and collected into 300ul of RNAlater (Ambion Inc, Austin, Texas) prior to DNA extraction (DNeasy Blood and Tissue Kit, Qiagen) from individual gills. qPCR analysis was performed using a Taqman assay developed for the detection of P. perurans $\mathrm{DNA}^{5}$.

Total RNA extraction. Following the removal of the whole, 2nd left gill from RNALater, Total RNA was extracted using the RNeasy Mini Plus Kit (Qiagen, Germany) according to the manufacturer's instructions. Briefly, $30 \mathrm{mg}$ gill tissue from each individual fish gill, irrespective of the presence of absence of mucoid lesions, was homogenized using a bead mill (Fisher Scientific) and $2.8 \mathrm{~mm}$ ceramic beads in $350 \mu \mathrm{l}$ RT lysis buffer and $1 \mu \mathrm{lDX}$ antifoam reagent (Qiagen, Germany), 5 pulses/sec, $10 \mathrm{~s}$, repeated 3 times. The optional DNase I (Qiagen, Germany) step was included to ensure complete elimination of gill genomic DNA. RNA was eluted in $70 \mu \mathrm{L}$ of nuclease -free water and stored at $-80^{\circ} \mathrm{C}$ until required. RNA was quantified using the Qubit RNA Assay Kit in Qubit 2.0 Fluorimeter (Thermo Fisher Scientific, USA). RNA integrity (RIN) was assessed using the RNA Nano 6000 Assay Kit of the Bioanalyzer 2100 system (Agilent Technologies CA, USA). Total RNA with RIN $\geq 8.0$ or higher were used for library construction.

Library construction and transcriptome sequencing. Library construction and transcriptome sequencing was outsourced (Novogene, Cambridge, UK). Total RNA from 6 control fish ( 0 dpi) and 6 fish from 4, 7, 14 and 16 dpi dpi was used for the construction of 30 RNA sequencing libraries using NEBNext Ultra RNA Library Prep Kit for Illumina (NEB, USA) according to manufacturer's instructions. Index codes were added to attribute sequences to each sample. The clustering of the index-coded samples was performed on a cBot Cluster Generation System using HiSeq PE Cluster Kit cBot-HS (Illumina) according to the manufacturer's instructions. After cluster generation, library preparations were sequenced on an Illumina Hiseq 2000 platform and 125 bp/150 bp paired-end reads were generated. FastqQc (Version 0.11.8) (www.bioinformatics.babraham.ac. $\mathrm{uk} /$ projects/fastqc/2) was utilised for quality assessment of reads from each sample and Multiqc (Version 1.7$)^{52}$ was used to visualise all FastQc results. No samples were identified as being bad quality, and all samples were included in the next step of the workflow process. Trimmomatic (v0.36) ${ }^{53}$ was used to trim paired reads in FASTQ files, using default parameters for paired-end mode and a minimum read length of $50 \mathrm{bp}$.

Differential expression analysis. Differential expression analysis was performed on total RNA from 6 fish from 4, 7, 14 and 16 dpi using the DESeq2 (Version 1.24.0) ${ }^{54}$ where each time point was compared to 6 fish from time 0 , naïve, pre-AGD samples. DESeq2 provided statistical routines for determining differential expression in digital gene expression data using a model based on the negative binomial distribution. The resulting $p$ values were adjusted to control for the false discovery rate (FDR $)^{55}$. Genes with an adjusted $p$-value ( $p$-adj) of $<0.05$ were determined to be differentially expressed. No minimum $\log _{2}$ FC threshold was assigned as a cut-off value for differential expression. Four lists of DEGs were generated: T0 vs T4 (4 dpi), T0 vs T5 (7 dpi), T0 vs T8 (14 dpi) and T0 vs T9 (16 dpi) down. Each list was further divided into down-regulated and up-regulated genes resulting in a total of eight DEGs lists. Volcano plots were created to visualise the DEGs at individual time points (Fig. 2).

Read mapping to the Atlantic salmon (Salmo salar) reference genome. The Salmo salar genome ICSASG_v2 (GenBank:GCF_000233375.1) ${ }^{23}$ was used to map the reads (Supplementary Table S1 online). Mapping was implemented using HiSat2 (version 2.1.0) (6) $^{56}$ using default parameters and paired-end mode. Counts were generated using featureCounts (v1.6.0 $)^{57}$ using the default parameters for paired-end reads. RNA-seq specific QC, sample correlation and visualisation were implemented using Seqmonk (Version 1.45.1) (https://www. bioinformatics.babraham.ac.uk/projects/seqmonk/2.

Principal component analysis (PCA) plots were generated to visualise the relationships between all samples, as well as samples by time point. The similarity between samples from thirty RNA-seq libraries ( 6 fish $\times 5$ groups) was analysed (Supplementary Fig. S1 online). An additional T0 group ( 6 individual fish) was sent for sequencing as an added precaution as this time point was considered critical to the success of the study. The RNA sequencing from all six fish in both control groups (T0_Ctrl, T0_AGD) was similar with regard to the total number of reads per fish and also in the total number of reads mapped (T0 Ctrl: 58312820 with $88.42 \%$ mapped, T0 AGD: (preinoculation) 56224414 with $88.03 \%$ mapped). A pairwise comparison between T0_Ctrl and T0_AGD identified only 1 DE gene between the 2 groups. Subsequently, the T0_GCRL fish $(n=6)$ were selected for the pairwise analysis of the RNA-seq data to the other time points.

Pathway and process enrichment analysis. Gene Ontology (GO $)^{58,59}$ and Kyoto Encyclopedia of Genes and Genomes (KEGG) ${ }^{60}$ pathway mapping was performed using Metscape ${ }^{24}$. The zebrafish (Danio rerio) database was used to determine GO enrichment as the option to use Salmo salar was not available in Metscape. 
For each given gene list in the DEG dataset, the following ontology sources were used: KEGG Functional Sets, KEGG Pathway, GO Biological Processes, GO Cellular Components, GO Molecular Functions, KEGG Structural Complexes and Reactome Gene Sets. A user-supplied list of 5815 protein coding genes, identified as $D$. rerio homologs, was used as the enrichment background. Terms with a $p$-value $<0.01$, a minimum count of 3 , and an enrichment factor $>1.5$ (the enrichment factor is the ratio between the observed counts and the counts expected by chance) were collected and grouped into clusters based on their membership similarities. More specifically, $p$-values were calculated based on the accumulative hypergeometric distribution ${ }^{61}$, and $q$-values were calculated using the Benjamin-Hochberg procedure to account for multiple testings ${ }^{55}$. Kappa scores ${ }^{62}$ were $^{2}$ used as the similarity metric when performing hierarchical clustering on the enriched terms, and sub-trees with a similarity of $>0.3$ were considered a cluster. The most statistically significant term within a cluster was chosen to represent the cluster.

Protein-Protein interaction (PPI). PPI was carried out using STRING (version 11.0, https://string-db. org) to generate protein association networks based on function for immune genes identified as enriched in KEGG pathways and Reactome gene sets. The default settings were used and Danio rerio selected as the species of interest as STRING does not provide Salmo salar as a species option.

Validation of RNA-seq data using Real-time PCR. Total RNA ( $1 \mu \mathrm{g})$ from each individual fish gill sample at 5 time points ( $0 \mathrm{dpi}, 4 \mathrm{dpi}, 7 \mathrm{dpi}, 14 \mathrm{dpi}$ and $16 \mathrm{dpi}$ ) was reverse transcribed to complementary DNA (cDNA) using the GoScript kit (Promega) as per manufacturer's instructions. Real-time PCR was performed using 48.48 Dynamic Array Integrated Fluidic Circuit (IFC) chips on the Biomark HD system (Fluidigm, USA). Ten genes differentially regulated in the gill RNA-seq data were selected including acod1: aconitate decarboxylase 1 (also known as irg1), cath1: cathelicidin 1, clra: c type lectin receptor a, cc4: c-c motif chemokine 4-like, lect2: leukocyte cell-derived chemotaxin 2-like, il-8: interleukin-8-like, il17a/f1: interleukin-17a/f1-like, nos: nitric oxide synthase 2, inducible, ptx: pentraxin-related protein-like, sap: serum amyloid P-component-like. Elongation factor 1 alpha (ef1a) was selected as the most appropriate housekeeping gene ${ }^{63}$. Gene primers were designed using PrimerQuest (Integrated DNA Technologies, https://eu.idtdna.com/). All primers were designed to have Tm of $62{ }^{\circ} \mathrm{C}$ and to be run in the qPCR at $58 \mathrm{C}$. Primer sequence details are provided in Supplementary Table S8 online.

A pre-amplification multiplex step was carried out on the target genes using a MiniAmp Plus PCR machine (Applied Biosystems) using the Preamp Master Mix (Cat. No. 100-5581, Fluidigm) as per manufacturer's instructions. The pre-amplified cDNA was treated with Exonuclease I to remove unincorporated primers prior to running on the IFC chip. The PCR assay mix consisted of $0.7 \mu \mathrm{L}$ of $50 \mathrm{mM}$ primer mix (IDT, Belgium), $3.5 \mu \mathrm{L}$ of $2 \mathrm{X}$ assay loading reagent (Fluidigm) and $2.8 \mu \mathrm{L}$ of $1 \mathrm{X}$ DNA elution buffer (Qiagen, Germany). The sample premix was prepared with $200 \mu \mathrm{L}$ of $2 X$ SsoFast EvaGreen supermix with low ROX (Bio-Rad, München, Germany) and $20 \mu \mathrm{L}$ of 20X Binding Dye Sample Loading Reagent (GE 48.48 Dynamic Array ${ }^{\text {ta }}$ Sample \& Assay Loading Reagent Kit, Item 85000821 , Fluidigm), of which $3.85 \mu \mathrm{L}$ of the sample pre-mix was combined with $3.15 \mu \mathrm{L}$ of the diluted pre-amplified PCR product for each sample inlet of the IFC chip. The PCR program was $95^{\circ} \mathrm{C}$ for $60 \mathrm{~s}, 30$ cycles of $95^{\circ} \mathrm{C}$ for $5 \mathrm{~s}, 58^{\circ} \mathrm{C}$ for $20 \mathrm{~s}$ followed by a melt curve protocol of $55^{\circ} \mathrm{C}$ to $95{ }^{\circ} \mathrm{C}$ with a ramp rate of $1{ }^{\circ} \mathrm{C} / 3 \mathrm{~s}$. Each sample was run in triplicate/chip and 3 chips used per validation. Melt curve analysis was preformed to confirm the specificity of the amplified PCR product. PCR amplification efficiency (E) was calculated for each gene of interest and the housekeeping gene by the generation of standard curves using tenfold serial dilutions of the cDNA template (standard curve: $\mathrm{R}^{2}>0.980$, amplification efficiency range 90-105\%). Melt curve analysis was used to confirm the amplification of single, PCR products. The relative expression for each gene was calculated using the delta delta CT (ddCT) method ${ }^{64}$ by the Real-Time PCR Analysis software on the Biomark HD (Fluidigm, USA).

Statistical analysis. For pathway and process enrichment analyses, $p$-values $<0.01$ were calculated based on the accumulative hypergeometric distribution, and $q$-values calculated using the Benjamin-Hochberg correction for multiple testing using Metascape. Kappa scores were used as the similarity metric when performing hierarchical clustering on the enriched terms, and sub-trees with a similarity of $>0.3$ were considered a cluster. The most statistically significant term within a cluster was chosen to represent the cluster. For qPCR analysis, the fold change of each gene at each time point was analysed relative to the T0 control using an un-paired t-test with differences considered significant at $p<0.05$. Ggplot2_3.2.1 in R studio Version 3.5.1 was used to generate the Spearman correlation data.

\section{Data availability}

All data supporting this study are included in the results section, the Supplementary material section online and openly available from the NCBI Geo Database, Accession Number GSE179972.

Received: 6 January 2021; Accepted: 23 September 2021

Published online: 19 October 2021

\section{References}

1. Munday, B. L. Diseases of Salmonids: Proceedings of the Workshop on Diseases of Australian fish and shellfish (Department of Agriculture and Rural Affairs, 1986).

2. Oldham, T., Rodger, H. \& Nowak, B. F. Incidence and distribution of amoebic gill disease (AGD)-An epidemiological review. Aquaculture 457, 35-42 (2016)

3. Adams, M. B. \& Nowak, B. F. Amoebic gill disease: Sequential pathology in cultured Atlantic salmon, Salmo salar L.. J. Fish Dis. 26, 601-614 (2003). 
4. Marcos-López, M. \& Rodger, H. D. Amoebic gill disease and host response in Atlantic salmon (Salmo salar L.): A review. Parasite Immunol. 42, 1-2 (2020).

5. Downes, J. et al. A longitudinal study of amoebic gill disease on a marine Atlantic salmon farm utilising a real-time PCR assay for the detection of Neoparamoeba perurans. Aquac. Environ. Interact. 7, 239-251 (2015).

6. Taylor, R. S. et al. Gill observations in Atlantic salmon (Salmo salar, L.) during repeated amoebic gill disease (AGD) field exposure and survival challenge. Aquaculture 290, 1-8 (2009).

7. Roberts, S. D. \& Powell, M. D. Freshwater bathing alters the mucous layer of marine Atlantic salmon Salmo salar L. J. Fish Biol. 72, 1864-1870 (2008).

8. Król, E. et al. Integration of transcriptome, gross morphology and histopathology in the gill of sea farmed Atlantic Salmon (Salmo salar): Lessons from multi-site sampling. Front. Genet. 11, 610 (2020).

9. Morrison, R. N. et al. Transcriptome profiling the gills of amoebic gill disease (AGD)-affected Atlantic salmon (Salmo salar L.): A role for tumor suppressor p53 in AGD pathogenesis?. Physiol. Genom. 26, 15-34 (2006).

10. Wynne, J. W. et al. Transcriptome analyses of amoebic gill disease-affected Atlantic salmon (Salmo salar) tissues reveal localized host gene suppression. Mar. Biotechnol. 10, 388-403 (2008).

11. Young, N. D., Cooper, G. A., Nowak, B. F., Koop, B. F. \& Morrison, R. N. Coordinated down-regulation of the antigen processing machinery in the gills of amoebic gill disease-affected Atlantic salmon (Salmo salar L.). Mol. Immunol. 45, 2581-2597 (2008).

12. Benedicenti, O., Collins, C., Wang, T., McCarthy, U. \& Secombes, C. J. Which Th pathway is involved during late stage amoebic gill disease?. Fish Shellfish Immunol. 46, 417-425 (2015).

13. Bridle, A. R., Morrison, R. N., Cupit Cunningham, P. M. \& Nowak, B. F. Quantitation of immune response gene expression and cellular localisation of interleukin-1 $\beta$ mRNA in Atlantic salmon, Salmo salar L., affected by amoebic gill disease (AGD). Vet. Immunol. Immunopathol. 114, 121-134 (2006).

14. Marcos-López, M. et al. Gene expression analysis of Atlantic salmon gills reveals mucin 5 and interleukin 4/13 as key molecules during amoebic gill disease. Sci. Rep. 8, 1-15 (2018).

15. Morrison, R. N. et al. Molecular cloning and expression analysis of tumour necrosis factor- $\alpha$ in amoebic gill disease (AGD)-affected Atlantic salmon (Salmo salar L.). Fish Shellfish Immunol. 23, 1015-1031 (2007).

16. Pennacchi, Y., Leef, M. J., Crosbie, P. B. B., Nowak, B. F. \& Bridle, A. R. Evidence of immune and inflammatory processes in the gills of AGD-affected Atlantic salmon, Salmo salar L. Fish Shellfish Immunol. 36, 563-570 (2014).

17. Pennacchi, Y., Adams, M. B., Nowak, B. F. \& Bridle, A. R. Immune gene expression in the gills of Atlantic salmon (Salmo salar L.) following experimental reinfection with Neoparamoeba perurans. Aquaculture 464, 410-419 (2016).

18. Boison, S. A., Gjerde, B., Hillestad, B., Makvandi-Nejad, S. \& Moghadam, H. K. Genomic and transcriptomic analysis of amoebisc gill disease resistance in Atlantic salmon (Salmo salar L.). Front. Genet. 10, 68 (2019).

19. Robledo, D., Hamilton, A., Gutiérrez, A. P., Bron, J. E. \& Houston, R. D. Characterising the mechanisms underlying genetic resistance to amoebic gill disease in Atlantic salmon using RNA sequencing. BMC Genom. 21, 1-11 (2020).

20. Marcos-López, M. et al. Oxidative stress is associated with late-stage amoebic gill disease in farmed Atlantic salmon (Salmo salar L). J. Fish Dis. 41, 383-387 (2018).

21. Morrison, R. N. et al. AGD pathogenesis?. Physiol. Genom. 26, 15-34 (2006).

22. Robledo, D., Gutiérrez, A. P., Barría, A., Yáñez, J. M. \& Houston, R. D. Gene expression response to sea lice in Atlantic salmon skin: RNA sequencing comparison between resistant and susceptible animals. Front. Genet. 9, 287 (2018).

23. Lien, S. et al. The Atlantic salmon genome provides insights into rediploidization. Nature 533, 200-205 (2016).

24. Karnovsky, A. et al. Metscape 2 bioinformatics tool for the analysis and visualization of metabolomics and gene expression data. Bioinformatics 28, 373-380 (2012).

25. Newton, K. \& Dixit, V. M. Signaling in innate immunity and inflammation. Cold Spring Harb. Perspect. Biol. 4, a006049 (2012).

26. McCormack, M., Dillon, E., O'connor, I. \& Maccarthy, E. Investigation of the initial host response of Naïve Atlantic Salmon (Salmo salar) inoculated with Paramoeba perurans. Microorganisms 9, 1-20 (2021).

27. Bayne, C. J. \& Gerwick, L. The Acute Phase Response and Innate Immunity of Fish. Developmental \& Comparative Immunology Vol. 25 (Elsevier, 2001).

28. Bridle, A. R., Davenport, D. L., Crosbie, P. B. B., Polinski, M. \& Nowak, B. F. Neoparamoeba perurans loses virulence during clonal culture. Int. J. Parasitol. 45, 575-578 (2015).

29. Schurch, N. J. et al. How many biological replicates are needed in an RNA-seq experiment and which differential expression tool should you use?. RNA 22, 839-851 (2016).

30. Michelucci, A. et al. Immune-responsive gene 1 protein links metabolism to immunity by catalyzing itaconic acid production. Proc. Natl. Acad. Sci. U. S. A. 110, 7820-7825 (2013).

31. Wu, R., Chen, F., Wang, N., Tang, D. \& Kang, R. ACOD1 in immunometabolism and disease. Cell. Mol. Immunol. 17, 822-833 (2020).

32. Vodovotz, Y. et al. Regulation of transforming growth factor $\beta 1$ by nitric oxide. Cancer Res. 59, 2142-2149 (1999).

33. Bystry, R. S., Aluvihare, V., Welch, K. A., Kallikourdis, M. \& Betz, A. G. B cells and professional APCs recruit regulatory T cells via CCL4. Nat. Immunol. 2, 1126-1132 (2001).

34. Chen, J., Lu, X. J., Yang, H. Y. \& Shi, Y. H. An interaction between a C-type lectin receptor and leukocyte cell-derived chemotaxin 2 of ayu, Plecoglossus altivelis. Fish Shellfish Immunol. 28, 245-248 (2010).

35. Gurung, P. \& Kanneganti, T. D. Immune responses against protozoan parasites: A focus on the emerging role of Nod-like receptors. Cell. Mol. Life Sci. 73, 3035-3051 (2016).

36. Botwright, N. A., Mohamed, A. R., Slinger, J., Lima, P. C. \& Wynne, J. W. Host-parasite interaction of Atlantic salmon (Salmo salar) and the Ectoparasite Neoparamoeba perurans in Amoebic Gill Disease. Front. Immunol. 12, 1-24 (2021).

37. Yamamoto, Y. \& Gaynor, R. B. IкB kinases: Key regulators of the NF-кB pathway. Trends Biochem. Sci. 29, 72-79 (2004).

38. Angot, A., Vergunst, A., Genin, S. \& Peeters, N. Exploitation of eukaryotic ubiquitin signaling pathways by effectors translocated by bacterial type III and type IV secretion systems. PLoS Pathog. 3, 0001-0013 (2007).

39. Tang, Q., Chakraborty, S. \& Xu, G. Mechanism of vaccinia viral protein B14-mediated inhibition of IkB kinase $\beta$ activation. J. Biol. Chem. 293, 10344-10352 (2018).

40. Yoshida, H., Nakaya, M. \& Miyazaki, Y. Interleukin 27: A double-edged sword for offense and defense. J. Leukoc. Biol. 86, 1295-1303 (2009).

41. Tsai, H. C., Velichko, S., Hung, L. Y. \& Wu, R. IL-17A and Th17 cells in lung inflammation: An update on the role of Th17 cell differentiation and IL-17R signaling in host defense against infection. Clin. Dev. Immunol. 2013, 1-12 (2013).

42. Ishigame, H. et al. Differential roles of Interleukin-17A and -17F in host defense against mucoepithelial bacterial infection and allergic responses. Immunity 30, 108-119 (2009).

43. Lee, Y., Clinton, J., Yao, C. \& Chang, S. H. Interleukin-17D promotes pathogenicity during infection by suppressing CD8 T Cell activity. Front. Immunol. 10, 1172 (2019).

44. Goriely, S., Neurath, M. F. \& Goldman, M. How microorganisms tip the balance between interleukin-12 family members. www.nature. com/reviews/immunol (2008).

45. Niedbala, W. et al. IL-35 is a novel cytokine with therapeutic effects against collagen-induced arthritis through the expansion of regulatory T cells and suppression of Th17 cells. Eur. J. Immunol. 37, 3021-3029 (2007). 
46. Deshmukh, H. S. et al. Metalloproteinases mediate mucin 5 AC expression by epidermal growth factor receptor activation. Am. J. Respir. Crit. Care Med. 171, 305-314 (2005).

47. Sheng, Y. H. et al. The MUC13 cell-surface mucin protects against intestinal inflammation by inhibiting epithelial cell apoptosis. Gut 60, 1661-1670 (2011).

48. Maher, D. M., Gupta, B. K., Nagata, S., Jaggi, M. \& Chauhan, S. C. Mucin 13: Structure, function, and potential roles in cancer pathogenesis. Mol. Cancer Res. 9, 531-537 (2011).

49. LaMonte, G. M. et al. Dual RNA-seq identifies human mucosal immunity protein Mucin-13 as a hallmark of Plasmodium exoerythrocytic infection. Nat. Commun. 10, 1-13 (2019).

50. Kilkenny, C., Browne, W., Cuthill, I. C., Emerson, M. \& Altman, D. G. Animal research: Reporting in vivo experiments: The ARRIVE guidelines. Br. J. Pharmacol. 160, 1577-1579 (2010).

51. Crosbie, P. B. B., Bridle, A. R., Cadoret, K. \& Nowak, B. F. In vitro cultured Neoparamoeba perurans causes amoebic gill disease in Atlantic salmon and fulfils Koch's postulates. Int. J. Parasitol. 42, 511-515 (2012).

52. Ewels, P., Magnusson, M., Lundin, S. \& Käller, M. MultiQC: Summarize analysis results for multiple tools and samples in a single report. Bioinformatics 32, 3047-3048 (2016).

53. Bolger, A. M., Lohse, M. \& Usadel, B. Trimmomatic: A flexible trimmer for Illumina sequence data. Bioinformatics 30, 2114-2120 (2014).

54. Love, M. I., Huber, W. \& Anders, S. Moderated estimation of fold change and dispersion for RNA-seq data with DESeq2. Genome Biol. 15, 1-21 (2014).

55. Hochberg, Y. \& Benjamin, Y. More powerful procedures for multiple significance testing. Stat. Med. 9, 811-818 (1990).

56. Kim, D., Langmead, B. \& Salzberg, S. L. HISAT: A fast spliced aligner with low memory requirements. Nat. Methods 12, 357-360 (2015).

57. Liao, Y., Smyth, G. K. \& Shi, W. FeatureCounts: An efficient general purpose program for assigning sequence reads to genomic features. Bioinformatics 30, 923-930 (2014).

58. Carbon, S. et al. The Gene Ontology resource: Enriching a GOld mine. Nucleic Acids Res. 49, D325-D334 (2021).

59. Ashburner, M. et al. Gene ontology: Tool for the unification of biology. Nat. Genet. 25, 25-29 (2000).

60. Kanehisa, M. \& Goto, S. KEGG: Kyoto encyclopedia of genes and genomes. Nucleic Acids Res. 28, 27-30 (2000).

61. Zar, J. H. Biostatistical Analysis (NJ Prentice Hall, 1999).

62. Cohen, J. A coefficient of agreement for nominal scales. Educ. Psychol. Meas. 20, 27-46 (1960).

63. Olsvik, P. A., Lie, K. K., Jordal, A. E. O., Nilsen, T. O. \& Hordvik, I. Evaluation of potential reference genes in real-time RT-PCR studies of Atlantic salmon. BMC Mol. Biol. 6, 1-9 (2005).

64. Livak, K. J. \& Schmittgen, T. D. Analysis of relative gene expression data using real-time quantitative PCR and the $2^{-\Delta \Delta C T}$ method. Methods 25, 402-408 (2001).

\section{Acknowledgements}

This material is based upon research supported by the Department of Agriculture, Food and the Marine under the Grant award No. 15 S 745.

\section{Author contributions}

E.M., I.O.C. L.M., J.C. conceived the project. A.T. and L.M. contributed to the design of the experiments. A.T., G.M., L.G., L.P., performed the experiments, contributed to the collection and analysis of data. A.T., L.G., L.P. conducted statistical analysis. A.T. and E.M. wrote the paper. All authors edited the paper.

\section{Competing interests}

The authors declare no competing interests.

\section{Additional information}

Supplementary Information The online version contains supplementary material available at https://doi.org/ 10.1038/s41598-021-99996-1.

Correspondence and requests for materials should be addressed to A.T.

Reprints and permissions information is available at www.nature.com/reprints.

Publisher's note Springer Nature remains neutral with regard to jurisdictional claims in published maps and institutional affiliations.

(c) (i) Open Access This article is licensed under a Creative Commons Attribution 4.0 International License, which permits use, sharing, adaptation, distribution and reproduction in any medium or format, as long as you give appropriate credit to the original author(s) and the source, provide a link to the Creative Commons licence, and indicate if changes were made. The images or other third party material in this article are included in the article's Creative Commons licence, unless indicated otherwise in a credit line to the material. If material is not included in the article's Creative Commons licence and your intended use is not permitted by statutory regulation or exceeds the permitted use, you will need to obtain permission directly from the copyright holder. To view a copy of this licence, visit http://creativecommons.org/licenses/by/4.0/.

(C) The Author(s) 2021 